\title{
Anomalous elastic buckling of layered crystalline materials in the absence of
}

\section{structure slenderness}

\author{
Manrui Ren ${ }^{1}$, Yilun Liu ${ }^{2}$, Jefferson Zhe Liu ${ }^{3 *}$, Lifeng Wang ${ }^{4}$, and Quanshui Zheng ${ }^{1 *}$ \\ ${ }^{1}$ Department of Engineering Mechanics, Center for Nano and Micro Mechanics, and Applied Mechanics Lab, \\ Tsinghua University, Beijing 100084, China \\ ${ }^{2}$ State Key Laboratory for Strength and Vibration of Mechanical Structures, School of Aerospace Engineering, \\ Xi'an Jiaotong University, Xi' an 710049, China \\ ${ }^{3}$ Department of Mechanical and Aerospace Engineering, Monash University, Clayton, VIC 3800, Australia \\ ${ }^{4}$ Department of Mechanical Engineering, Stony Brook University, Stony Brook, NY 11794-2300, United \\ States
}

Email: zhe.liu@monash.edu; zhengqs@tsinghua.edu.cn

Phone: +61-3-99053627

\begin{abstract}
Layered crystalline materials, such as graphene, boron nitride, tungsten sulfate, phosphorene, etc., have attracted enormous attentions, due to their unique crystal structures and superior mechanical, thermal, and physical properties. Making use of mechanical buckling is a promising route to control their structural morphology and thus tune their physical properties, giving rise to many novel applications. In this paper, we employ molecular dynamics (MD) simulations and theoretical modeling to study the compressive buckling of a column made of layered crystalline materials with the crystal layers parallel to the compressive direction. We find that the mechanical buckling of the layered crystalline materials exhibits two anomalous and counter-intuitive features as approaching the zero slenderness ratio. First, the critical buckling strain $\varepsilon_{\mathrm{cr}}$ has a finite value that is much lower than the material's elastic limit strain. A continuum mechanics model (by homogenizing the layered materials) is proposed for the $\varepsilon_{\mathrm{cr}}$, which agrees well with the results of MD simulations. We find that the $\varepsilon_{\mathrm{cr}}$ solely depends on elastic constants without any structural dimension, which appears to be an intrinsic material property and thus is defined as intrinsic buckling strain (IBS), $\begin{gathered}\mathrm{IBS} \\ \mathrm{cr}\end{gathered}$ in this paper. Second, below a certain nanoscale length, $l_{0}$, in the compressive direction (e.g., about 20 $\mathrm{nm}$ for graphite), the critical buckling strain $\varepsilon_{\mathrm{cr}}$ shows a size effect, i.e., increasing as the column length $L$ decreases. To account for the size effect, inspired by our recently developed multi-beam shear model (Liu et al., 2011), a bending energy term of individual crystal layer is introduced in our continuum model. The theoretical model of $\varepsilon_{\text {cr }}$ agrees well with the size effects observed in MD
\end{abstract}


simulations. This study could lay a ground for engineering layered crystalline materials in various nano-materials and nano-devices via mechanical buckling.

Keywords: Layered crystalline materials; Intrinsic buckling strain; Size effect; Shear buckling mode; Extreme elasticity anisotropy

\section{Introduction}

Buckling, as a mechanical instability, is a common phenomenon in nature (Gere and Timoshenko, 1998; Price and Cosgrove, 1990; Zartman and Shvartsman, 2010). It is often treated as a nuisance to be avoided. This view is changing with the growing knowledge of this phenomenon (Biot, 1957; Bowden et al., 1998; Brau et al., 2010; Budd et al., 2003; Efimenko et al., 2005; Gere and Timoshenko, 1998; Hohlfeld and Mahadevan, 2011; Huang et al., 2005; Hunt et al., 2000; Kim et al., 2011; Pocivavsek et al., 2008; Wadee et al., 2004) and the emerging successful cases of employing mechanical buckling in real applications (Efimenko et al., 2005; Guo et al., 2011; Kim et al., 2008; Kim et al., 2009; Kim et al., 2010; Koo et al., 2010; Rogers et al., 2010; Stafford et al., 2004; Y. Wang et al., 2011; Zang et al., 2013). For example, utilizing buckled interconnecting components in electronic devices leads to "stretchable electronics" that can accommodate large stretching and compressive loads without breaking (Kim et al., 2008; Rogers et al., 2010). Mechanical buckling of a thin stiff film on a soft substrate under an in-plane compression can alter the surface morphology and thus modulate the surface physicochemical properties, giving rise to various applications, such as artificial skins (Efimenko et al., 2005), micro-devices to measure mechanical properties of thin polymer and nanoparticle films (Leahy et al., 2010; Stafford et al., 2004), dynamically controlled surface wettability (Zang et al., 2013), enhancement of light extracting efficiency from organic light-emitting diodes (Koo et al., 2010), and dynamic display of biomolecule micropatterns (Kim et al., 2009). The surface ripples also have many applications in micro-fluidic devices (Efimenko et al., 2005) and artificial muscle actuators (Zang et al., 2013).

The discovery of graphene (Novoselov et al., 2004) has stimulated intensive research interests for two dimensional crystalline materials, such as $\mathrm{BN}, \mathrm{MoS}_{2}, \mathrm{WS}_{2}$, silicene, graphyne, and so on (Golberg et al., 2010; Malko et al., 2012; Nicolosi et al., 2013; Vogt et al., 2012; Wang et al., 2012; Wilson and Yoffe, 1969). For this class of materials, atoms distributed in a layered crystal lattice are 
bonded via strong chemical bonds, whereas different crystal layers interact with each other through weak van der Waals or electrostatic forces. Such a two dimensional crystalline material has a unique combination of structural, mechanical and physical properties, enabling great potentials for applications in electronic devices, catalysts, batteries, and super-capacitors, as seen in recent extensive experimental and theoretical studies (Geim and Novoselov, 2007). In practice, these materials are often fabricated in a form with multiple crystal layers stacked together, either for the convenience of fabrication or intentionally. For example, tuning either the number of layers or the stacking sequence of different types of crystal layers can modulate electronic properties of the resultant van der Waals heterostructures (Geim and Grigorieva, 2013; Haigh et al., 2012). It turns out that using multi-layers of graphene as a building block of graphene cellular foams is essential for the observed super-elasticity under a large compressive strain up to $80 \%$ in experiments (Qiu et al., 2012).

In addition to the widely studied approaches to tailor the physical properties of layered crystalline materials, e.g., scissoring graphene into different shapes (Ci et al., 2008), chemical doping (Ci et al., 2010), chemical or physical adsorption (Elias et al., 2009; Nair et al., 2010; Schedin et al., 2007; Xu et al., 2009), mechanical buckling caused by a compressive load parallel to the basal planes can serve as a promising method to enable new applications. There are already several successful experimental studies. It has been reported that the reversible mechanical buckling of a stack of graphene-oxide layers is the origin for the hydration responsive property of graphene oxide liquid crystal in experiments (Guo et al., 2011). The periodically rippled graphene ribbons formed on a pre-stretched elastomer substrate can be used as high performance strain sensors (Wang et al., 2011). A super-hydrophobic surface with a reversibly tunable wettability has been realized using crumpled graphene films (Zang et al., 2013). However, employing mechanical buckling of layered crystalline materials in applications is still hampered by inadequate understanding of this phenomenon.

The most well-known elastic buckling is the bending mode of instability studied back to Euler's era (Gere and Timoshenko, 1998). For a slender structure, such as a beam, plate, or thin film, being subject to a longitudinal compression, lateral deflection will occur beyond a critical load. This is because bending is energetically less costly than compression for these slender structures. Most of the applications described previously are based on this type of instability. It should be noted that the unique atomistic structures of layered materials, i.e., strong in-plane covalent chemical bonds, giving 
rise to a very high in-plane elastic modulus, and weak out-of-plane van der Waals or electrostatic interactions, yielding a very small interlayer shear modulus, imply that the interlayer shear effect plays an important role in the mechanical behaviors of layered materials (Ghosh and Arroyo, 2013; Li et al., 2007; Liu et al., 2001; Liu et al., 2011; Yu, 2004). For the layered materials, above a critical compressive load along in-plane directions, shear mode instability occurs. The shear deformation among adjacent atomic layers generates a lateral displacement and then releases the compressive strain. Such a shear mode of instability was observed in wood (Byskov et al., 2002), fiber reinforce composites (Budiansky et al., 1998; Kyriakides et al., 1995), and geological strata (Price and Cosgrove, 1990). However, there are very few experimental and theoretical studies for the shear mode instability of the layered crystalline solids (Cranford, 2013; Liu et al., 2010; Ren et al., 2015), particularly in terms of the critical buckling load. It should be noted that the internal buckling of anisotropic medium was theoretically studied previously, as reviewed in Biot's book (Biot, 1965) and a recent book from Bažant (Bažant and Cedolin, 2010). But there are two limitations. First, these previous theoretical studies are continuum mechanics models and the validation of continuum modeling to the layered crystalline materials at nanoscale is ambiguous. Second, the previous theoretical studies of internal buckling usually assumed the anisotropic materials incompressible (Bažant and Cedolin, 2010; Biot, 1965), which is inconsistent to most layered crystalline materials.

In this paper, we firstly employ molecular dynamics (MD) simulations to simulate the mechanical buckling of graphite, hexagonal boron nitride ( $h$-BN), virtual graphite, and virtual $h$-BN (i.e., parameters of Lennard-Jones potential used to describe the interlayer van der Waals interaction are altered to tune the interlayer shear modulus) at a nanometer scale in section 2 . In section 3 , a continuum mechanics model is developed to describe the buckling behaviors of a column with an infinite width (i.e., zero slenderness ratio) made of layered crystalline materials. In this model, the layered materials are assumed as homogenous medium of transversely isotropic elasticity. This continuum mechanics model provides accurate predictions for the critical buckling strain $\varepsilon_{\mathrm{cr}}$ down to a column length $L$ of about $20 \mathrm{~nm}$ in MD simulations. A theoretical model for a more general case (orthorhombic crystalline symmetry) is presented in appendix. Section 4 presents a continuum model to explain the observed size effect of the $\varepsilon_{\mathrm{cr}}$ in MD simulations. A length parameter $l_{0}$ is defined. In case of the column length $L$ being larger than $l_{0}$, the size effect is becoming negligible. Section 5 discusses the implications of such elastic buckling in layered crystalline materials and its potential 
applications. Conclusions are drawn in section 6.

\section{MD simulations}

In light of great potentials of utilizing mechanical buckling in nano devices (Koo et al., 2010; Rogers et al., 2010; Zang et al., 2013), it is of great interests to investigate this phenomenon at a nanometer scale for the layered crystalline materials. In this section, non-equilibrium molecular dynamics (NEMD) simulations is employed to study the elastic buckling of the hexagonal layered crystalline materials, e.g. graphite, $h$-BN, virtual-graphite, and virtual $h$-BN columns with a length $L$ down to $2 \mathrm{~nm}$.

Figure 1(a) depicts our molecular system: a graphite column composed of periodic A/B stacked graphene layers (normal to the $z$-axis) with their basal planes parallel to the longitudinal axis (the $x$-axis) and the transverse axis (the $y$-axis). Longitudinal length $L$ is selected between $2 \mathrm{~nm}$ and $40 \mathrm{~nm}$. Periodic boundary conditions (PBC) are applied in all three coordinate directions. The dashed box in Fig. 1(a) represents the super-cell used in our MD simulations. The super-cell is compresed in the $x$ direction at a constant velocity which varies from $10^{-5}$ to $1 \AA / \mathrm{ps}$ in different simulations, meanwhile the $y$ and $z$ dimensions of the super-cell are fixed and the three super-cell vectors remain perpendicular to each other through the simulations. The PBC along the $x$-axis restricts wavelength of the first-order buckling as $L$. The PBC in the $y$ and $z$ directions result in an infinite radius of gyration $\rho$ of the cross-section (i.e., $\rho \sim \infty$ ). Thus our molecular models have a ratio of slenderness $L / \rho=0$.

NEMD simulations are performed using the LAMMPS code (Plimpton, 1995). The adaptive intermolecular reactive empirical bond order (AIREBO) potential (Stuart et al., 2000) is adopted to describe the interatomic interactions of the graphite column. Temperature of the whole system is fixed at $0.1 \mathrm{~K}$ using the Berendsen thermostat, with the temperature calculated after removing the center-of-mass velocity. A time step of $1.0 \mathrm{fs}$ is used and the simulations are continued until the elastic buckling took place. Although the interlayer delamination has been observed in the post-buckling of multilayer graphene (Cranford, 2013), in this work we just care about the critical buckling strain and the interlayer delamination does not occur for all of the cases in our MD simulations.

For a column with $L=4.6 \mathrm{~nm}$, Fig. 1(c) shows the results of potential energy and stress $\sigma_{x}$ as functions of compressive strain $\varepsilon_{x}$ in the $x$ direction, at two different loading velocities $10^{-4} \AA / \mathrm{ps}$ and $10^{-2} \AA / p s$. The strain is defined as $\varepsilon_{x}=\delta / L$, where $\delta$ is the change of the unit cell in $x$-dimension. The 
results of potential energy and the stress in the $x$-direction can be directly output from LAMMPS. In the beginning, both potential energy results appear as a parabolic function of the strain $\varepsilon_{x}$. This is consistent to the obtained linear stress-strain relations in Fig. 1(c). At the critical point, the energy curves start to deviate from the parabolic relation and accordingly the stress curves exhibit a significantly drop, indicating the happening of mechanical buckling. Indeed, the carbon atoms exhibit a clear lateral displacement in the $z$ direction after the critical point. Color-map in Fig. 1(a) shows the relative magnitude of lateral displacement obtained at a loading velocity of $10^{-4} \AA / \mathrm{ps}$, in which the blue color denotes a relatively larger displacement than the red color. Figure 1(b) sketches the buckling shape. Figure 1(c) shows that the determined critical compressive strain value $\varepsilon_{\mathrm{cr}}$ sensitively depends on the loading velocity. At $10^{-2} \AA / p s$, the $\varepsilon_{\text {cr }}$ equals to $0.250 \%$, whereas at a lower loading velocity of $10^{-4} \AA / p s$ the critical strain result significantly reduces to $\varepsilon_{\mathrm{cr}}=0.124 \%$. This is a common phenomenon in dynamic buckling (Lindberg, 2003). After release of the compressive load, the buckled graphite column bounces back, fully recovering its original shape.

(a)

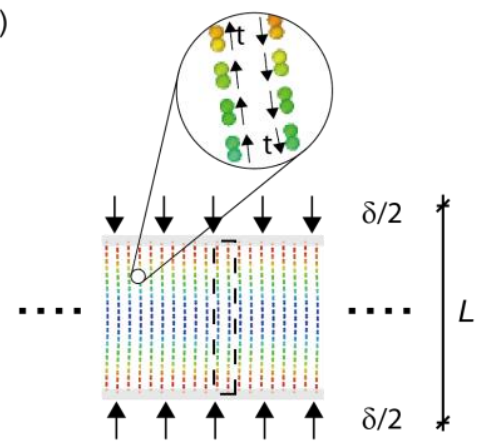

(b)

(c)

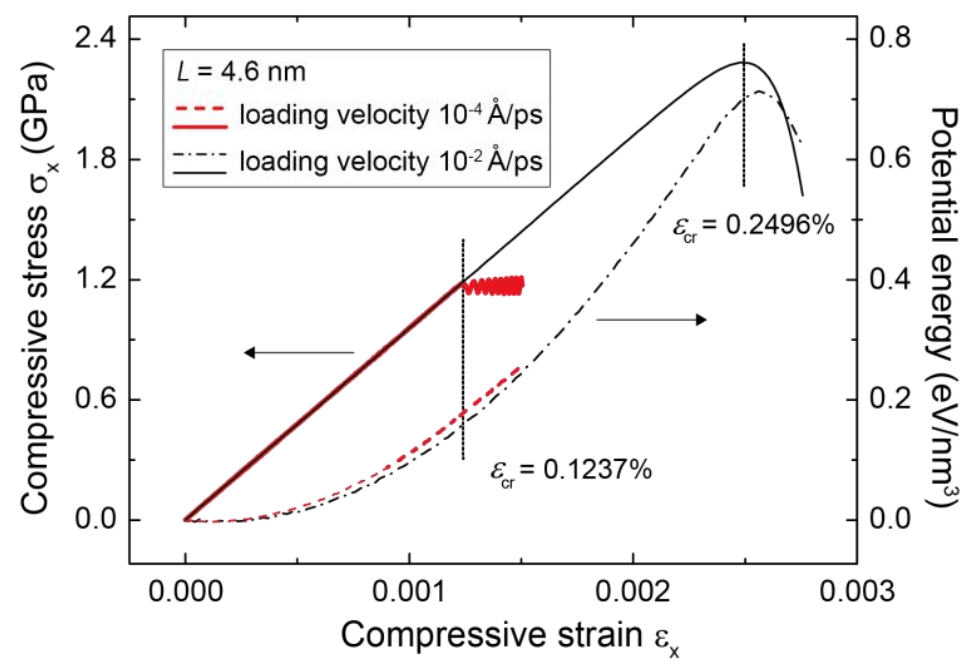

Fig. 1. (a) Molecular model for mechanical buckling of a graphite column in NEMD simulations. Graphene basal planes are parallel to the $x-y$ plane. The box enclosed by dashed lines represents the super-cell. Periodic 
boundary conditions are applied in all three directions. Note that the periodic boundary conditions applied in lateral directions imply an infinite gyration radius $\rho$ and thus lead to a zero slenderness ratio $L / \rho$. The super-cell is compressed in $x$ direction at a constant velocity which varies from $10^{-5}$ up to $1 \AA$ ps in different simulations, meanwhile the $y$ and $z$ dimensions of the super-cell are fixed and the three super-cell vectors remain perpendicular to each other through the simulations. The color map illustrates relative magnitude of the lateral displacement $(z)$ after mechanical buckling, in which the blue color denotes a larger displacement than the red color. (b) A sketch of the buckling shape. (c) Potential energy and stress $\sigma_{x}$ versus compressive strain $\varepsilon_{\square}$ in MD simulations. At the critical point, the potential energy and stress results show an abrupt change, indicating the happening of mechanical buckling.

Figure 2 summarizes the dependence of $\varepsilon_{\mathrm{cr}}$ on the loading velocity for a graphite column with different length $L$ from about $2 \mathrm{~nm}$ to $40 \mathrm{~nm}$. Reduction of the loading velocity generally leads to a decrease of obtained $\varepsilon_{\mathrm{cr}}$ values. Apparently, a convergence is achieved below $10^{-4} \AA / \mathrm{ps}$. A close inspection of the data in Table I indicates the difference of $\varepsilon_{\mathrm{cr}}$ is indeed very small for loading velocity of $10^{-4} \AA / \mathrm{ps}$ and $10^{-5} \AA / \mathrm{ps}$. In addition, it is found that a shorter column has a higher convergent $\varepsilon_{\mathrm{cr}}$ result. Above $L \sim 20 \mathrm{~nm}$, only a minor difference is observed in the $\varepsilon_{\mathrm{cr}}$ value at loading velocity of $10^{-4}$ $\AA / p s$, i.e., between $0.038 \%$ and $0.034 \%$. In the following, we will denote the critical strain determined for a graphite column with length $L \geq 20 \mathrm{~nm}$ and at a loading velocity $\leq 10^{-4} \AA$ A $/ \mathrm{ps}$ in MD simulations as the converged $\varepsilon_{\text {cr. }}$

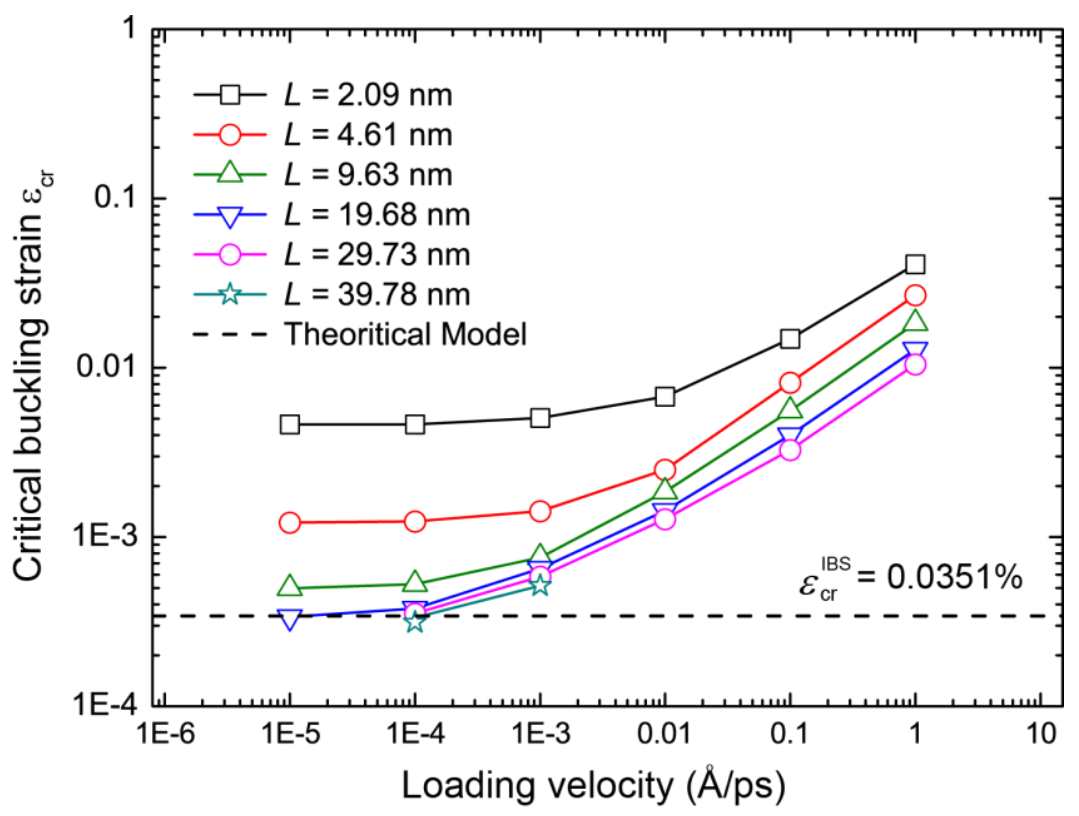

Fig. 2. Critical elastic buckling strain $\varepsilon_{\mathrm{cr}}$ of a graphite column (Fig. 1(a)) as a function of loading velocity in 
NEMD simulations. Results for different longitudinal length $L$ from $2.09 \mathrm{~nm}$ to $39.78 \mathrm{~nm}$ are shown. Note that the PBC in $y$ and $z$ directions result in an infinite radius of gyration $\rho$ of the cross-section (i.e., $\rho \sim \infty$ ). Thus all MD models have a ratio of slenderness $L / \rho=0$. Prediction from the continuum mechanics model Eq. (12) in section 3 is shown as the dashed line for a comparison.

Table I. The critical buckling strain $\varepsilon_{\mathrm{cr}}$ of a graphite column with different length $L$ under a (low) loading velocity of $10^{-4}$ or $10^{-5} \AA / \mathrm{ps}$.

\begin{tabular}{ccccccc}
\hline $\boldsymbol{L} \mathbf{( n m})$ & $\mathbf{2 . 0 9}$ & $\mathbf{4 . 6 1}$ & $\mathbf{9 . 6 3}$ & $\mathbf{1 9 . 6 8}$ & $\mathbf{2 9 . 7 3}$ & $\mathbf{3 9 . 7 8}$ \\
\hline $\mathbf{1 0}^{-\mathbf{4}} \mathbf{\AA} / \mathbf{p s}$ & $0.464 \%$ & $0.124 \%$ & $0.0525 \%$ & $0.0378 \%$ & $0.0353 \%$ & $0.0334 \%$ \\
$\mathbf{1 0}^{-5} \stackrel{\AA}{\mathrm{A}} / \mathbf{p s}$ & $0.464 \%$ & $0.122 \%$ & $0.0498 \%$ & $0.0337 \%$ & - & - \\
\hline
\end{tabular}

The NEMD simualtions were also carried out for a column made of $h$-BN, some virtual graphite, and virtual $h$-BN (similar to Fig. 1(a)) to determine their critical buckling strain. The virutal material models were created by artificially changing the parameter $\varepsilon$ or $\sigma$ in the LJ potential that were used to describe the interlayer van der Waals interaction. Table II lists the parameters of the original LJ potentials. Given that the predicted $C_{44}$ value from the AIREBO force field for graphite is far smaller than the experimental results (discussed later), the value of $\varepsilon$ is increased by 2 to 10 times (Table III). The in-plane interaction of a graphene layer or $h$-BN layer is described using the Tersoff potentials (Kınac1 et al., 2012; Tersoff, 1989). To avoid the dynamic effect, we adotped a low loading velocity of $10^{-5} \AA / p s$. Note that for the graphite columns, the results of $\varepsilon_{\text {cr }}$ determined using Tersoff and LJ potentials are similar to those using AIREBO potential (Table III). A similar dependence of $\varepsilon_{\mathrm{cr}}$ on the column length $L$ is observed in Fig. 3. As $L$ increases above $\sim 20 \mathrm{~nm}$, the critical buckling strain approaches to a converged value $\varepsilon_{\mathrm{cr}}$. 


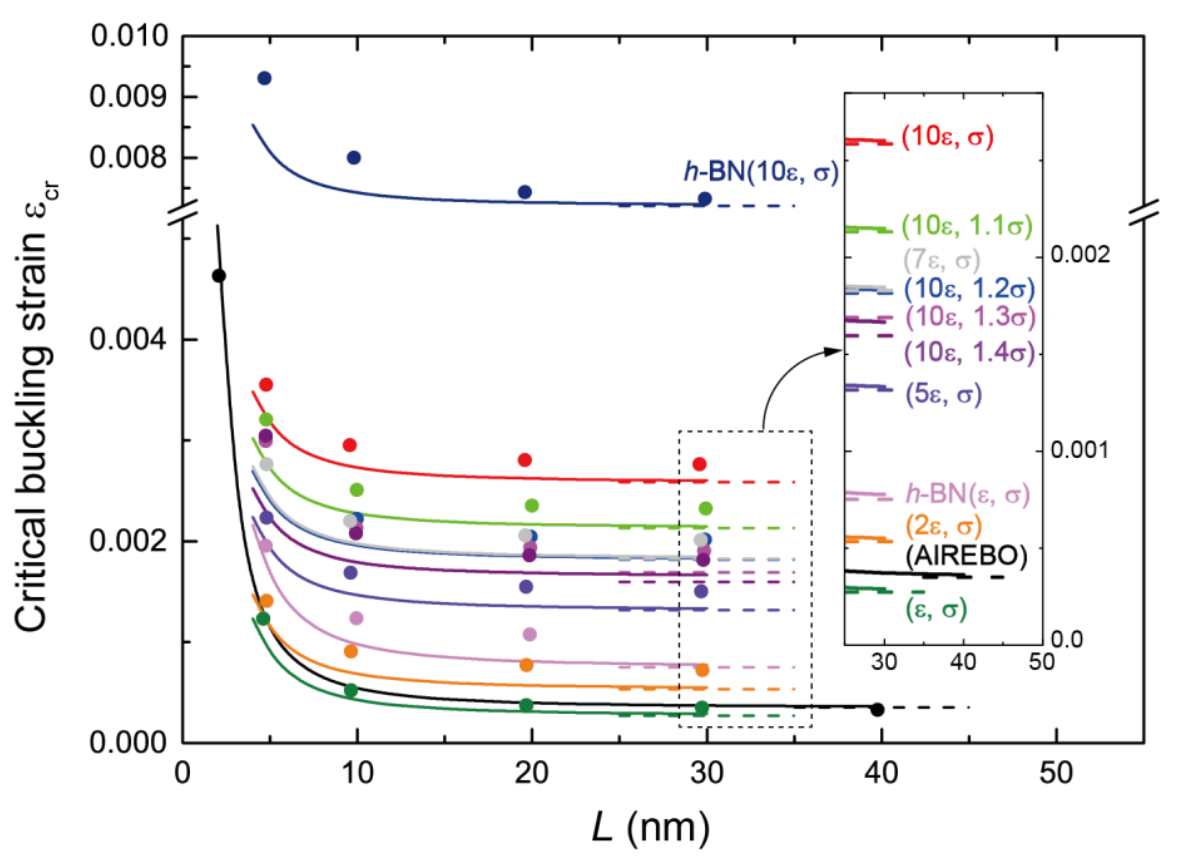

Fig. 3. The critical buckling strain results of a column made of different layered materials as a function of column length $L$ determined in MD simulations using Tersoff and LJ potentials. Different materials are represented by their corresponding LJ paprameters $(\varepsilon, \sigma)$ and the notations without " $h$-BN" represent (virtual) graphite by default (cf. Table III). Results using AIREBO potential for graphite are also included for comparison. To avoid the dynamic effect, a low loading velocity of $10^{-5} \AA / \mathrm{ps}$ is adopted. Note that the PBC in $y$ and $z$ directions results in an infinite radius of gyration $\rho$ of the cross-section and thus a zero slenderness ratio for all MD models. The predictions of the continuum theoretical model Eq. (12) in section 3 are shown as dashed lines. The solid lines represent the theoretical model Eq. (18) in section 4, which is developed to account for the observed size effect in MD.

Table II. Parameters of the Lennard-Jones potential used to describe the interlayer van der Waals interactions for graphite and $h$-BN in our NEMD simulations.

\begin{tabular}{cccc}
\hline Atom pair & $\varepsilon(\mathrm{meV})$ & $\sigma(\AA)$ & References \\
\hline C-C & 2.84 & 3.40 & (Stuart et al., 2000) \\
B-B & 4.116 & 3.45 & (Baowan and Hill, 2007) \\
N-N & 6.281 & 3.365 & (Baowan and Hill, 2007) \\
B-N & 5.085 & 3.41 & (Baowan and Hill, 2007) \\
\hline
\end{tabular}

Table III summarises the converged $\varepsilon_{\mathrm{cr}}$ values for all different material models at $L=19.68 \mathrm{~nm}$ and a loading velocity of $10^{-5} \AA / \mathrm{ps}$. Interestingly, the buckling strain values are lower than $1 \%$, which is far lower than the mechanical strength of graphene and $h$-BN (Lee et al., 2008; Song et al., 2010). This suggests such a mechanical buckling is, in principle, achievable in experiments. More 
interestingly, this mechanical buckling phenomenon takes place at a zero slenderness ratio, which is believed to be highly unlikely for conventional isotropic materials. For the virtual materials, increasing the parameter $\varepsilon$ of the LJ potential (i.e., enhancing interlayer binding strength) leads to a higher converged $\varepsilon_{\mathrm{cr}}$ value, whereas increasing the parameter $\sigma$ in the LJ potential (i.e., enlarging the equilibrium interlayer distance) results in a lower converged $\varepsilon_{\mathrm{cr}}$ value. This observation will be explained in section 3 .

Table III. A comparison of the converged $\varepsilon_{\mathrm{cr}}$ results from NEMD simulations ( $L=19.69 \mathrm{~nm}$ and low loading velocity of $10^{-5} \AA / \mathrm{ps}$ ) and our theoretical model ${ }_{\text {cr }}^{\text {IBS }}$ [Eq. (12)]. The calculated elastic modulus $C_{11}$ and $C_{44}$ are shown as well.

\begin{tabular}{|c|c|c|c|c|}
\hline L-J parameter & $C_{11}(\mathrm{GPa})$ & $\boldsymbol{C}_{\mathbf{4 4}}(\mathrm{GPa})$ & $\begin{array}{c}\text { Continuum } \\
\text { mechanics model }\end{array}$ & MD simulation \\
\hline Graphite $^{\mathrm{a}}$ & 980 & 0.3442 & $0.03512 \%$ & $0.03370 \%$ \\
\hline Graphite $(\varepsilon, \sigma)^{\mathrm{b}}$ & 1221 & 0.3329 & $0.02727 \%$ & $0.03482 \%$ \\
\hline Virtual Graphite $(2 \varepsilon, \sigma)^{\mathrm{b}}$ & 1248 & 0.6671 & $0.05343 \%$ & $0.06115 \%$ \\
\hline Virtual Graphite $(5 \varepsilon, \sigma)^{\mathrm{b}}$ & 1273 & 1.677 & $0.1317 \%$ & $0.1425 \%$ \\
\hline Virtual Graphite $(7 \varepsilon, \sigma)^{\mathrm{b}}$ & 1288 & 2.3563 & $0.1829 \%$ & $0.1899 \%$ \\
\hline Virtual Graphite $(10 \varepsilon, \sigma)^{\mathrm{b}}$ & 1309 & 3.3844 & $0.2586 \%$ & $0.2656 \%$ \\
\hline Virtual Graphite $(10 \varepsilon, 1.1 \sigma)^{\mathrm{b}}$ & 1204 & 2.5678 & $0.2133 \%$ & $0.2359 \%$ \\
\hline Virtual Graphite $(10 \varepsilon, 1.2 \sigma)^{\mathrm{b}}$ & 1110 & 2.0176 & $0.1817 \%$ & $0.2048 \%$ \\
\hline Virtual Graphite $(10 \varepsilon, 1.3 \sigma)^{\mathrm{b}}$ & 1035 & 1.7493 & $0.1691 \%$ & $0.1912 \%$ \\
\hline Virtual Graphite $(10 \varepsilon, 1.4 \sigma)^{\mathrm{b}}$ & 971 & 1.5497 & $0.1596 \%$ & $0.1863 \%$ \\
\hline$h-\mathbf{B N}^{\mathrm{c}}$ & 832 & 0.6254 & $0.07518 \%$ & $0.1082 \%$ \\
\hline Virtual $h-\mathrm{BN}(10 \varepsilon, \sigma)^{\mathrm{c}}$ & 884 & 6.3725 & $0.7211 \%$ & $0.7441 \%$ \\
\hline
\end{tabular}

\footnotetext{
${ }^{\mathrm{a}}$ modeled by AIREBO force field.

${ }^{\mathrm{b}}$ modeled by Tersoff and LJ potentials. The value of parameter $\varepsilon$ in LJ potential is increased by 2, 5,

7, and 10 times, respectively and the value of parameter $\sigma$ in LJ potential is increased by $1.1,1.2,1.3$, and 1.4 times, respectively. Before the NEMD simulations and the calculations for $C_{44}$, interlayer distances and C-C bond lengths are optimized to remove residual stresses.

${ }^{\mathrm{c}}$ modeled by Tersoff and LJ potentials. The value of parameter $\varepsilon$ in LJ potential is increased by 1 and 10 times, respectively. Before the NEMD simulations and the calculations for $C_{44}$, interlayer distances and B-N bond lengths are optimized to remove residual stresses.
}

According to the continuum mechanics models derived in sections 3 and 4, the interlayer shear modulus and the elastic modulus in the compressive direction, namely $C_{44}$ and $C_{11}$, are the determinant parameters for the converged $\varepsilon_{\mathrm{cr}}$ value. These two moduli should be calculated using the same force fields adopted in MD simulations. The $C_{11}$ modulus is determined by fitting the potential energy curve as a fucntion of strain $U=1 / 2 C_{11} \varepsilon_{x}^{2}$ prior to the buckling. For graphite [Fig. 1(b)], it 
gives a value of $980 \mathrm{GPa}$ that is close to the experimental result $1060 \mathrm{GPa}$ (Kelly, 1981). Results for the virtual graphites, $h$-BN, and virtual $h$-BN are shown in Table III.

The shear modulus $C_{44}$ was calculated using an in-house FORTRAN code. A simple shear deformation was applied to an A/B stacked 11-layers-graphene or $h$-BN (with periodic boundary conditions along the two directions of basal plane). That is, each layer is kept rigid and displaced with respect to each other in a direction parallel to the basal plane. The shear displacement is a linear function of the layer's position (out-of-plane direction) in the stack. Coordinates of the atoms in the sheared graphite were then fed into the in-house FORTRAN code that calculated LJ potential energy of the $6^{\text {th }}$ layer with the same cut-off distance as used in the LAMMPS simulations. The magnitude of shear strains $\gamma$ were selected to ensure the deformation is within a linear elastic region, i.e., $-0.003<\gamma$ $<0.003$. The obtained LJ potential energy of the graphene or $h$-BN layer in the middle of the stack exhibits a nearly perfect parabolic relation with respect to $\gamma$. For graphite, fitting the results using $U=$ $1 / 2 C_{44} \gamma^{2}$ yielded the shear modulus $C_{44}=0.3442 \mathrm{GPa}$.

This value is significantly smaller than the experimental results: 4.3-4.5 GPa (Kelly, 1981; Tan, 2012a) and first-principles density functional theory (DFT) calculation results: 3.9-4.5 GPa (Mounet and Marzari, 2005). The book from Kelly (Kelly, 1981) has provided a review on elastic properties of graphite measured using different experimental techniques. They concluded that neutron scattering experiments gave the most reliable $C_{44}$ value, $4.6 \pm 0.2 \mathrm{GPa}$, because it is also consistent with the specific heat data. Recently Tan et.al. (Tan, 2012b) used Raman spectroscopy to study the phonon properties of high quality few layer graphene flakes and concluded that the $C_{44}$ is around $4.3 \mathrm{GPa}$, where structural defects should be minor. The DFT simulations were carried out for a perfect graphite crystal (Mounet and Marzari, 2005). Thus these experiments and first-principles calcualtions indicate that the reported and widely accepted $C_{44}$ value $\sim 4.5 \mathrm{GPa}$ should arise from the intrinsic van der Waals interaction among graphene layers in bulk graphite. Our $C_{44}$ result was also calculated for a perfect graphite crystal but using empirical force fields. In these force fields, the Lennard-Jones potential $U_{\mathrm{LJ}}(r)=4 \varepsilon\left[(\sigma / r)^{12}-(\sigma / r)^{6}\right]$ is used to describe the interlayer van der Waals interations. The two free parameters $\varepsilon$ and $\sigma$ were fitted to reproduce two experimental results: interlayer distance $3.4 \AA$ and elastic modulus $C_{33}=36.5 \mathrm{GPa}$ in $z$ direction. Thus the $\mathrm{LJ}$ force field often provides unsatisfactory predictions of other physical properties, e.g., binding energy between graphene layers and cleavage energy of graphite (Gould et al., 2013; Lebègue et al., 2010; Liu et al., 2012; Sorella et al., 2009). We believe that the observed big difference of our calculated shear modulus $C_{44}$ from other experiments 
and first-principles calculations should originate from the same reason. The $C_{44}$ results for other materials are listed in Table III as well. Generally, the increasing of LJ potential parameter $\varepsilon$ will significantly increase the interlayer shear modulus $C_{44}$.

\section{Continuum mechanics model of intrinsic buckling strain}

In this section, a continuum mechanics model is developed for the critical elastic buckling strain of a column made of the layered materials with a zero slenderness ratio and under a quasi-static loading. Here, the layered materials are modeled as homogenous mediums with hexagonal crystal symmetry (i.e., transversely isotropic elastic). This is because graphite and $h$-BN have such crystal symmetry. Theoretical model for a more general case - orthorhombic symmetry - is presented in Appendix. Figure 1(b) depicts our coordinate system, in which the basal planes of the crystal layers are parallel to the $x-y$ plane. The origin point is placed in the center of the column and the $y-z$ plane overlaps with the middle cross-section plane. A periodic boundary condition is applied in the lateral direction ( $z$-axis), yielding $\rho \rightarrow \infty$ and thus $L / \rho=0$. This system is modeled as a plane-strain problem (in the $x$-z plane), aiming to be consistent with some popular experimental setups, i.e., utilizing strain mismatch between a film made of layered crystalline materials and a pre-stretched substrate to drive the elastic buckling (Bowden et al., 1998; Efimenko et al., 2005; Kim et al., 2011; Koo et al., 2010; Zang et al., 2013) or bending induced periodic stripe/kinking microstructures during mechanical peeling of graphite flakes (Liu et al., 2010). Given that the $z$-axis in our model is the vertical direction of the film/substrate system in experiments, a compressive strain (via release of the pre-stretch deformation of substrate) is applied along the basal plane direction ( $x$-axis). Under the constraint from the substrate, deformation in $y$ direction is negligible, leading to the plane-strain condition.

For the column model shown in Fig. 1(b), we use the tensor index notation $x_{1}=x, x_{2}=y, x_{3}=z$. Thus, the displacement $\left(u_{i}\right)$ and stress $\left(\sigma_{i j}\right)$ boundary conditions are

$$
\begin{aligned}
& \left.u_{1}\right|_{x=L / 2}=-\delta / 2,\left.\quad \sigma_{12}\right|_{x=L / 2}=\left.\sigma_{13}\right|_{x=L / 2}=0, \\
& \left.u_{1}\right|_{x=-L / 2}=\delta / 2,\left.\quad \sigma_{12}\right|_{x=-L / 2}=\left.\sigma_{13}\right|_{x=-L / 2}=0, \\
& \left.u_{3}\right|_{x=L / 2}=\left.u_{3}\right|_{x=-L / 2},
\end{aligned}
$$

where $\delta$ is the relative displacement of the both ends moving toward each other. Note that the boundary conditions for displacements are consistent with those of the MD simulations, in which both ends are fixed. The displacement fields can be expressed as 


$$
u_{1}=x+{ }_{n=1}^{N} a_{n} \frac{L}{2 n} \sin \frac{2 n x}{L}, \quad u_{2}=0, \quad u_{3}=b_{0} z+{ }_{n=1}^{N} b_{n} \frac{L}{2 n} \cos \frac{2 n x}{L}
$$

where $\varepsilon=\delta / L$ represents the homogeneous compressive strain in the $x$-direction prior to elastic buckling. Clearly, $u_{1}$ is an odd function of coordinate $x$. Thus, a series composed of sine functions is used to represent the displacement after the elastic buckling. Note that $u_{1}$ is independent of $z$ because of the periodic boundary condition in the $z$ direction. The displacement $u_{2}$ is set as zero to be consistent to the plane strain condition discussed early. The displacement $u_{3}$ is an even function of coordinate $x$. It is thus expressed as a series made of cosine functions. The first term of $u_{3}$ accounts for the lateral expansion in the $z$-direction. The periodic boundary condition suggests such a lateral expansion should be uniform. Hence the first term is expressed as a linear function of the coordinate $z$. It is also natural to assume the lateral expansion linearly depends on the applied compressive strain $\varepsilon$ along the $x$-direction. The coefficient $b_{0}$ should be taken as a parameter that represents different types of boundary conditions in the z-direction. In our MD simulations, the lateral expansion is fixed. Therefore, the coefficient $b_{0}=0$. In the case of a freely relaxed boundary condition in $z$ direction, $b_{0}$ should be equal to $b_{0}=C_{13} / C_{33}$.

These displacement fields [Eq. (2)] satisfy the boundary conditions [Eq. (1)], with undetermined coefficients $a_{n}$ and $b_{n}$. Only when the load is above the critical buckling value $\delta_{\text {cr }}$, the $a_{n}$ and $b_{n}$ will have nonzero solutions.

Deformation gradient $\boldsymbol{F}$ and the Green strain $\boldsymbol{E}$ can be derived based on the displacement fields as

$$
\begin{aligned}
& F_{11}=1+\frac{u_{1}}{x}, \quad F_{33}=1+\frac{u_{3}}{z}, \quad F_{13}=\frac{u_{1}}{z}, \quad F_{31}=\frac{u_{3}}{x}, \\
& E_{11}=\left(F_{11}^{2}+F_{31}^{2} 1\right) / 2, \quad E_{33}=\left(F_{33}^{2}+F_{13}^{2} 1\right) / 2, \quad E_{13}=\left(F_{11} F_{13}+F_{31} F_{33}\right) / 2 .
\end{aligned}
$$

A hexagonal layered crystalline material has a transversely isotropic elasticity. In our model, since its basal plane is in the $x-y$ plane, the constitutive law of linear elasticity can be expressed as

$$
\left.\begin{array}{l}
{ }_{11} \\
22 \\
33 \\
23 \\
12
\end{array}\right)=\left(\begin{array}{ccccccc}
C_{11} & C_{12} & C_{13} & 0 & 0 & 0 \\
C_{12} & C_{11} & C_{13} & 0 & 0 & 0 \\
C_{13} & C_{13} & C_{33} & 0 & 0 & 0 \\
0 & 0 & 0 & C_{44} & 0 & 0 \\
0 & 0 & 0 & 0 & C_{44} & 0 \\
0 & 0 & 0 & 0 & 0 & \left(C_{11}\right. & \left.C_{12}\right) / 2
\end{array}\right)\left(\begin{array}{c} 
\\
11 \\
22 \\
33 \\
2_{23} \\
2_{31} \\
2_{12}
\end{array}\right)
$$


where $C_{i j}$ are the stiffness constants. For investigating instability problem, we use the Green strain $E_{i j}$ instead of the infinitesimal strain $\varepsilon_{i j}$ in order to take account nonlinearity and consequently we use the second Piola-Kirchhoff stress, $T_{i j}$, instead of the conventional stress $\sigma_{i j}$. Substituting Eq. (4) into Eq. (5) yields

$$
T_{11}=C_{11} E_{11}+C_{13} E_{33}, \quad T_{33}=C_{13} E_{11}+C_{33} E_{33}, \quad T_{13}=2 C_{44} E_{13} .
$$

Then the strain energy density $U$ is

$$
U=1 / 2\left(T_{11} E_{11}+2 T_{13} E_{13}+T_{33} E_{33}\right) .
$$

Integrating the energy density $U$ in the column leads to the potential energy as

$$
W=\frac{1}{L} \int_{-L / 2}^{L / 2} U d x,
$$

in which a unit length is taken along the $y$ and $z$ directions, owing to the plane strain condition and the periodic boundary condition, respectively.

Following the principle of minimum total potential energy, partial derivatives of $W$ with respect to the undetermined coefficients $a_{n}$ and $b_{n}$ should be equal to zero. Thus,

$$
\begin{aligned}
& g_{1}=\boldsymbol{W} / b_{1}=b_{1}\left(C_{44}\left(\begin{array}{llll}
C_{11} & 2 C_{44} b_{0} & \left.C_{13} b_{0}\right)
\end{array}\right) / 2+\mathrm{O}\left(b_{1}\left(C_{11}+C_{13} b_{0}^{2}+2 C_{44} b_{0}^{2}\right)^{2} / 4\right)=0\right. \\
& g_{2}=\boldsymbol{W} / b_{2}=b_{2}\left(C_{44}\left(\begin{array}{llll}
C_{11} & 2 C_{44} b_{0} & C_{13} b_{0}
\end{array}\right)\right) / 2+\mathrm{O}\left(b_{2}\left(C_{11}+C_{13} b_{0}^{2}+2 C_{44} b_{0}^{2}\right)^{2} / 4\right)=0 \\
& f_{1}=\boldsymbol{W} / a_{1}=a_{1}\left(C_{11}\left(\begin{array}{ll}
3 C_{11} & \left.b_{0} C_{13}\right)
\end{array}\right) / 2+\mathrm{O}\left(a_{1}\left(3 C_{11}+C_{13} b_{0}^{2}\right)^{2} / 4\right)=0\right. \\
& f_{2}=\boldsymbol{W} / a_{2}=a_{2}\left(C_{11}\left(3 C_{11} b_{0} C_{13}\right)\right) / 2+\mathrm{O}\left(a_{2}\left(3 C_{11}+C_{13} b_{0}^{2}\right)^{2} / 4\right)=0
\end{aligned}
$$

where the quadratic and higher order terms of strain $\varepsilon$ are omitted. Note that derivatives with respect to $b_{n}(n=1,2, \ldots)$ always yield the same equation as

$$
C_{44}-\left(C_{11}-2 C_{44} b_{0}-C_{13} b_{0}\right) \varepsilon=0 \text {. }
$$

The derivatives with respect to $a_{n}(n=1,2, \ldots)$ lead to another equation as

$$
C_{11}-\left(3 C_{11}-b_{0} C_{13}\right) \varepsilon=0 .
$$

From Eq. (10) or (11), we obtained the critical strain as

$$
{ }_{\mathrm{cr}}^{\mathrm{IBS}}=\frac{C_{44}}{C_{11}\left(2 C_{44}+C_{13}\right) b_{0}},
$$

or 


$$
{ }_{\mathrm{cr}}^{\mathrm{IBS}}=\frac{C_{11}}{3 C_{11} C_{13} b_{0}} .
$$

In our homogeneous continuum mechanics model, the displacement fields are expressed as infinite series that satisfy the boundary conditions, so that the solution Eq. (12) or Eq. (13) is the exact solution of the intrinsic buckling strain of the layered materials without slenderness. Note that Eq. (12) was firstly reported in the $\mathrm{PhD}$ thesis of one of the authors (Liu, 2002).

The prediction from Eq. (12) or (13) is valid only if the magnitude of $\varepsilon_{\mathrm{cr}}$ is small. Otherwise the higher order terms cannot be neglected in Eq. (9). From the aspect of physics, the adopted linear elasticity model may not be valid in the case of finite deformation. More importantly, only when the predicted $\varepsilon_{\mathrm{cr}}$ is lower than the material's elastic limit strain, the mechanical buckling could take place. Since Eq. (13) predicts a critical buckling strain $\varepsilon_{\mathrm{cr}}>1 / 3$, its prediction should not be considered reliable.

It is interesting to notice that the Eq. (12) only includes the elastic constants of materials without any structural dimensions suggesting that such a mechanical buckling is an intrinsic property of materials. It is reasonable to understand this feature because in the case of $L / \rho=0$, the column [Fig. 1(a)] is inherently "structure-less". Here we define the critical buckling strain at $L / \rho=0$ [Eq. (12)] as the intrinsic buckling strain (IBS) $\varepsilon_{\mathrm{cr}}^{\mathrm{IBS}}$. The parameter $n$ in displacement fields [Eq. (2)] represents different buckling modes. Interestingly, all the buckling modes share one degenerate eigenvalue, i.e., the $\varepsilon_{\mathrm{cr}}^{\mathrm{IBS}}$ in Eq. (12).

The value of $\varepsilon_{\mathrm{cr}}^{\mathrm{IBS}}$ for graphite is plotted in Fig. 2 (the dashed line) to compare with MD simulation results using the AIREBO force field. The value of $b_{0}$ should be zero to be consistent with the boundary conditions in MD simulations, as discussed before. Thus we obtain the IBS $\varepsilon_{\mathrm{cr}}^{\mathrm{IBS}}=$ $C_{44} / C_{11}=0.34416 / 980=0.03512 \%$, in which the $C_{11}$ modulus and $C_{44}$ modulus are the values calculated in section 2. Figure 2 shows that the theoretical IBS agrees with the converged $\varepsilon_{\mathrm{cr}}$ from MD simulations very well (for $L \geq 20 \mathrm{~nm}$ and under a loading velocity lower than $10^{-4} \AA / \mathrm{ps}$ ). The theoretical $\varepsilon_{\mathrm{cr}}^{\mathrm{IBS}}$ values are also compared with MD simualtion results for graphite, $h$-BN, virtual graphite, and virtual $h$-BN (with Tersoff and LJ force fields) in Fig. 3. For each case, the predicted $\varepsilon_{\mathrm{cr}}^{\mathrm{IBS}}$ agrees well with the converged $\varepsilon_{\mathrm{cr}}$ result in MD simulations. A detailed comparison is 
summarised in Table III and Fig. 4. We can, therefore, conclude that the IBS model accurately describes the critical elastic buckling strain of the studied layered crystalline mateirals at a zero slendness ratio, at a sufficiently large length scale $L$, under a quasi-static loading condition.

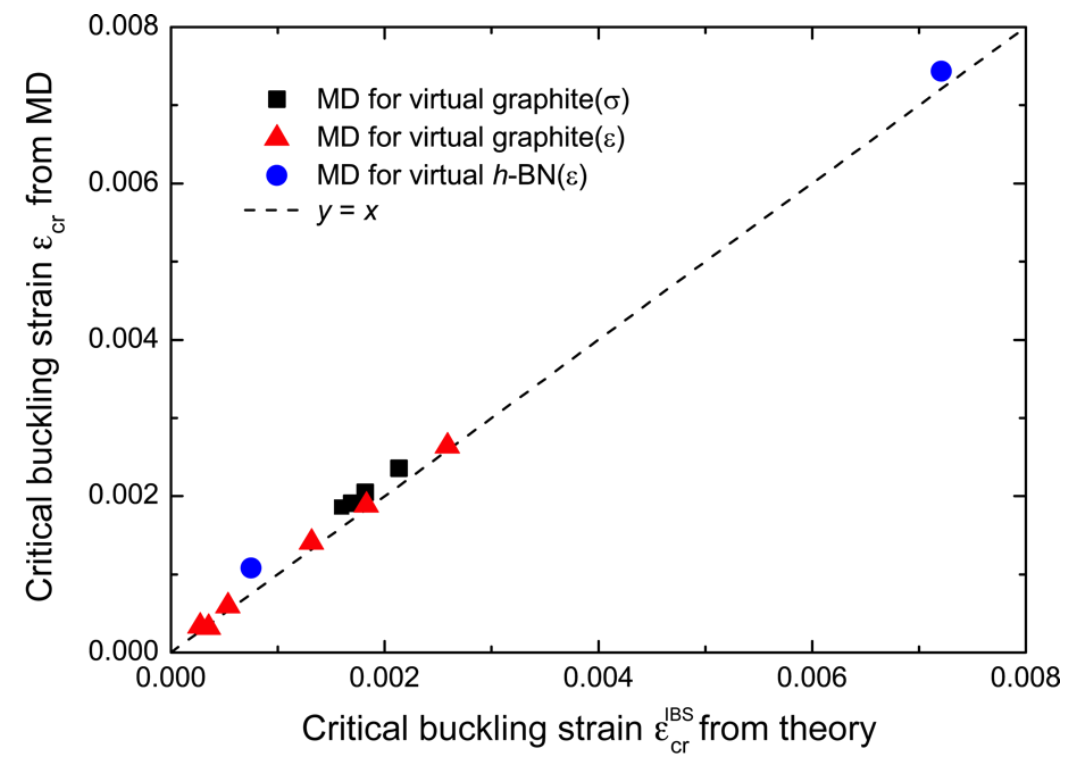

Fig. 4. A comparison of the intrinsic buckling strain $\underset{\mathrm{cr}}{\mathrm{IBS}}$ [Eq. (12) by setting $b_{0}=0$ ] with those converged $\varepsilon_{\mathrm{cr}}$ results from MD simulations. Each symbol represents one type of hexagonal layered crystalline materials simulated in section 2 .

Prior to ending this section, the theoretical IBS results under the freely relaxed side boundary condition $\left(b_{0}=C_{13} / C_{33}\right)$ of a list of hexagonal layered crystalline materials are calculated and shown in Table IV. These materials are selected based on a thorough survey done by Wang and Zheng for hexagonal crystal materials with an extreme elastic anisotropy degree (Wang and Zheng, 2007). Owing to the intrinsic layered atomic structures, most of the hexagonal layered crystalline materials have an in-plane elastic constant $C_{11}$ much larger than other elastic constants, particularly the shear modulus $C_{44}$. Thus, Eq. (12) often yields a smaller value of $\varepsilon_{\mathrm{cr}}^{\mathrm{IBS}}$ than that of Eq. (13), with only one exception in the materials that we visited, InSe, for which Eq. (13) leads to a smaller value. However, this predicted $\varepsilon_{\mathrm{cr}}^{\mathrm{IBS}}$ appears to be much higher than its material's yield strain and thus the elastic buckling is practically impossible. It is listed here just for theoretical interests. For those layered materials that attract enormous attentions at present, such as graphite, $h-\mathrm{BN}, \mathrm{MoS}_{2}$, and $\mathrm{WS}_{2}$, their $\varepsilon_{\mathrm{cr}}^{\mathrm{IBS}}$ results are smaller than $15 \%$. It is thus feasible to manipulate morphologies of these layered materials via the intrinsic buckling mode and thus tune their physical properties in experiments for 
novel applications. It should be noted that elastic constants in Table IV are from experiments. For graphite and $h$-BN, the results are different from Table III.

We note that critical strain $\varepsilon_{\mathrm{cr}}^{\mathrm{IBS}}$ predicted in Eq. (12) becomes $\varepsilon_{\mathrm{cr}}^{\mathrm{IBS}}=(1-v) /(1-2 v)$ for isotropic materials, where is the Poisson's ratio. Because of the thermodynamics restriction:

$11 / 2$, this critical value of strain would exceed elastic limit strains for most real materials, and therefore is impractical.

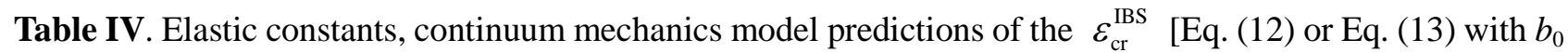
$\left.=C_{13} / C_{33}\right]$ of selected hexagonal layered crystalline materials with a high degree of elastic anisotropy. Most of the materials are adopted from Table I in reference (Wang and Zheng, 2007). Elastic constants of $h$-BN are from reference (Duclaux et al., 1992). Elastic constants of $\mathrm{WS}_{2}$ are from reference (Volkova et al., 2012).

\begin{tabular}{|c|c|c|c|c|c|c|}
\hline Materials & $C_{11}$ & $C_{12}$ & $C_{13}$ & $C_{33}$ & $C_{44}$ & $\begin{array}{l}\text { Continuum } \\
\text { mechanics } \\
\text { prediction }\end{array}$ \\
\hline Graphite (C) & 1060 & 180 & 15 & 36.5 & 4.5 & 0.0043 \\
\hline Molybdenum sulfide $\left(\mathrm{MoS}_{2}\right)$ & 238 & -54 & 23 & 51 & 18.6 & 0.0882 \\
\hline Biotite $\left[\mathrm{K}(\mathrm{Mg}, \mathrm{Fe})_{3} \mathrm{AlSi}_{3} \mathrm{O}_{10}(\mathrm{OH}, \mathrm{F})_{2}\right]$ & 186 & 32 & 12 & 54 & 5.8 & 0.0321 \\
\hline Phlogopite $\left[\mathrm{KMg}_{3} \mathrm{AlSi}_{3} \mathrm{O}_{10}(\mathrm{OH}, \mathrm{F})_{2}\right], \mathrm{B}$ & 178 & 30 & 15 & 51 & 6.5 & 0.0383 \\
\hline Phlogopite $\left[\mathrm{KMg}_{3} \mathrm{AlSi}_{3} \mathrm{O}_{10}(\mathrm{OH}, \mathrm{F})_{2}\right], \mathrm{A}$ & 179 & 32 & 26 & 51.7 & 5.6 & 0.0349 \\
\hline Muscovite $\left[\mathrm{KAl}_{2} \mathrm{Si}_{3} \mathrm{O}_{10}(\mathrm{OH}, \mathrm{F})_{2}\right]$ & 178 & 42.4 & 14.5 & 54.9 & 12.2 & 0.0727 \\
\hline Gallium sulfide (peizoel) $(\mathrm{GaS})$ & 126.5 & 35.7 & 14.3 & 41.6 & 12 & 0.1059 \\
\hline Gallium selenide (peizoel) $(\mathrm{GaSe})$ & 106.4 & 30 & 12.1 & 35.8 & 10.2 & 0.1069 \\
\hline Rubidium nickel chloride $\left(\mathrm{RbNiCl}_{3}\right)$ & 35.2 & 10.0 & 22 & 72.2 & 2.5 & 0.0927 \\
\hline Indium selenide (InSe) & 118.1 & 47.5 & 32 & 38.2 & 11.7 & 0.3606 \\
\hline Hexagonal Boron Nitride (h-BN) & 750 & 150 & - & 18.7 & 2.52 & 0.0034 \\
\hline Tungsten sulfide $\left(\mathrm{WS}_{2}\right)$ & 236 & 61 & 8 & 42 & 12 & 0.0522 \\
\hline
\end{tabular}

There are many interesting layered crystalline materials that have other types of crystal symmetry (Geim and Grigorieva, 2013). For example, the perovskite-type material $\mathrm{LaNb}_{2} \mathrm{O}_{7}$, $(\mathrm{Ca}, \mathrm{Sr})_{2} \mathrm{Nb}_{3} \mathrm{O}_{10}, \mathrm{Bi}_{4} \mathrm{Ti}_{3} \mathrm{O}_{12}$ and $\mathrm{Ca}_{2} \mathrm{Ta}_{2} \mathrm{TiO}_{10}$ have an orthorhombic symmetry. Thus a continuum mechanics model for an orthorhombic crystalline material is presented in Appendix. Note that for this symmetry, the column could undergo a mechanical buckling in either of the two lateral directions [i.e., $y$ or $z$-axis in Fig. 1(b)], in comparison with only one direction (i.e., z-axis) for the hexagonal crystalline materials. Consequently, there is one more result for the $\varepsilon_{\mathrm{cr}}^{\mathrm{IBS}}$. Please refer to Appendix for details. 


\section{Size effect of the critical buckling strain}

Figure 2 and Figure 3 demonstrate a significant size effect for the critical buckling strain of a column with length $L$ smaller than $20 \mathrm{~nm}$ (meanwhile the slenderness ratio equals to zero). Indeed, for a graphite column length $L$ reducing from $20 \mathrm{~nm}$ to $2 \mathrm{~nm}$, the magntiude of $\varepsilon_{\mathrm{cr}}$ increases from $0.03343 \%$ to $0.4635 \%$, i.e., more than 10 times increse. The continuum theoretical model presented in section 3 cannot explain this size-dependent phenomenon. In this section, we will extend the continuum model in section 3 to explain the observed size effect.

Careful analysis of the atomistic structures of the buckled layered crystalline materials reveals five types of deformation modes: (1) stretch/compression of the chemical bonds and (2) change of bond angles in the layer plane, (3) interlayer compression, (4) interlayer shear, and (5) bending of the dihedral bond angles out of the layer plane. In our continuum mechanics model for a homogeneous media (section 3), the strain term $E_{11}$ can describe deformation modes (1) and (2), the $E_{33}$ term accounts for mode (3), and the $E_{13}$ term corresponds to mode (4). It appears that the mode (5), i.e., the out-of-plane bending of each atomically thin layer, is not considered. Previous studies showed that the mechanical strain energy arising from the bending of atomically thin materials generally follows $U \sim \frac{1}{2} D_{b}{ }^{2}$, where $D_{b}$ is the bending stiffness and $\kappa$ is the curvature. For a bulk layered crystalline material, this energy term is negligible for two reasons. First, the value of $D_{b}$ is normally very small. Second, the deformation of a column under compression at a large size scale yields a small $\kappa$ value. However, when $L$ value reduces down to nanometer range, the $\kappa$ value in the buckled structure becomes more significant. The bending energy term, which accounts for the mode (5), should not be omitted anymore. It should be noted that the above bending energy term should be distinguished from the bending energy in the classic Euler or Timoshenko beam model, in which the bending effect arises from the stretch and compression on the opposite sides of a beam. We believe this is an inherent limitation of using classic continuum mechanics theory to describe the deformation of the layered crystalline materials at nanometer scale.

Then we add the bending energy term to Eq. (8):

$$
W=\frac{1}{L} \int_{-L / 2}^{L / 2} U d x+\frac{1}{h L} \int_{-L / 2}^{L / 2} \frac{1}{2} D_{b} \kappa^{2} d x
$$

where $h$ is interlayer space of the layered materials. The curvature can be approximated as $\kappa=\frac{\partial^{2} u_{3}}{\partial x^{2}}$. 
Based on the displacement fields in Eq. (2), the curvature can be expressed as

$$
={ }_{n=1}^{N} b_{n} \frac{2 n}{L} \cos \frac{2 n \quad x}{L} .
$$

The equilibrium state requires that the partial derivatives of $W$ with respect to the undetermined coefficients $a_{n}$ and $b_{n}$ should be equal to zero. Thus,

$$
\begin{aligned}
& g_{1}=\partial \boldsymbol{W} / \partial b_{1}=b_{1}\left(C_{44}-\left(C_{11}-2 C_{44} b_{0}-C_{13} b_{0}\right) \varepsilon+\frac{D_{b}(2 \pi)^{2}}{h L^{2}}\right)+\mathrm{O}\left(b_{1}\left(C_{11}+C_{13} b_{0}^{2}+2 C_{44} b_{0}^{2}\right) \varepsilon^{2} / 4\right)=0 \\
& g_{2}=\partial \boldsymbol{W} / \partial b_{2}=b_{2}\left(C_{44}-\left(C_{11}-2 C_{44} b_{0}-C_{13} b_{0}\right) \varepsilon+\frac{D_{b}(4 \pi)^{2}}{h L^{2}}\right)+\mathrm{O}\left(b_{2}\left(C_{11}+C_{13} b_{0}^{2}+2 C_{44} b_{0}^{2}\right) \varepsilon^{2} / 4\right)=0 \\
& \ldots . . \\
& f_{1}=\partial \boldsymbol{W} / \partial a_{1}=a_{1}\left(C_{11}-\left(3 C_{11}-b_{0} C_{13}\right) \varepsilon\right) / 2+\mathrm{O}\left(a_{1}\left(3 C_{11}+C_{13} b_{0}^{2}\right) \varepsilon^{2} / 4\right)=0 \\
& f_{2}=\partial \boldsymbol{W} / \partial a_{2}=a_{2}\left(C_{11}-\left(3 C_{11}-b_{0} C_{13}\right) \varepsilon\right) / 2+\mathrm{O}\left(a_{2}\left(3 C_{11}+C_{13} b_{0}^{2}\right) \varepsilon^{2} / 4\right)=0
\end{aligned}
$$

Note that adding the bending energy term in Eq. (14) leads to an extra term $\frac{D_{b}(2 n)^{2}}{h L^{2}}$ in Eq. (16) compared with Eq. (9). The critical buckling strain now becomes

$$
\varepsilon_{c r}=\frac{C_{44}}{C_{11}-2\left(C_{44}+C_{13}\right) b_{0}}+\frac{D_{b}}{\left(C_{11}-2\left(C_{44}+C_{13}\right) b_{0}\right) h}\left(\frac{2 n \pi}{L}\right)^{2} .
$$

By defining a length parameter $l_{0}=2 \sqrt{D_{b} / C_{44} h}$, the Eq. (17) can be written as

$$
\begin{aligned}
\varepsilon_{c r} & =\left[1+\left(n \frac{l_{0}}{L}\right)^{2}\right] \frac{C_{44}}{C_{11}-2\left(C_{44}+C_{13}\right) b_{0}} \\
& =\left[1+\left(n \frac{l_{0}}{L}\right)^{2}\right] \varepsilon_{c r}^{I B S}
\end{aligned}
$$

The difference between Eq. (18) and the IBS critical buckling strain in Eq. (12) is a geometry dependent pre-factor, where the size effect clearly manifests itself. For a large $L$ value, Eq. (18) reduces to the $\varepsilon_{\mathrm{cr}}^{\mathrm{IBS}}$ in Eq. (12). Reducing the column length $L$ leads to an enhancement of the critical buckling strain. In addition, Eq. (18) shows that the different buckling modes $n$ do not share a degenerated critical buckling strain value any more.

For the graphite column (AIREBO force field) in section 2, we have the parameters $D_{\mathrm{b}}=1.46 \mathrm{eV}$ (Singh et al., 2013), $C_{44}=0.3442 \mathrm{GPa}, h=0.3369 \mathrm{~nm}$. Thus the $l_{0}$ can be calculated as $8.9 \mathrm{~nm}$. The theoretical model [Eq. (18)], shown as the solid black line in Fig. 3, is compared with MD simulation 
results. The agreement is very good. For the cases of $h$-BN, graphite (Tersoff and LJ potentials), and virtual graphite, Table V lists the bending stiffness $D_{\mathrm{b}}$ results, the equilibrium interlayer distance $h$, and the calculated length parameter $l_{0}$. Figure 3 compares the theoretical model Eq. (18) with MD simulations of these cases as well. The agreement is also good, indicating the bending of atomic layers as origin of the observed size effect. Overall the $l_{0}$ value is quite small, varying from $2-10 \mathrm{~nm}$. Only when the column length $L$ (i.e., buckling wavelength) close or below $l_{0}$, the critical buckling strain will significantly deviates from the intrinsic buckling strain $\underset{\mathrm{cr}}{\mathrm{IBS}}$.

Table V. The bending stiffness $D_{\mathrm{b}}$, the equilibrium interlayer distance $h$, and the calculated length parameter $l_{0}$ of $h$-BN, graphite and virtual graphite are summarized.

\begin{tabular}{|c|c|c|c|}
\hline L-J parameter & $D_{\mathrm{b}}(\mathrm{eV})$ & $h(\mathrm{~nm})$ & $l_{0}(\mathrm{~nm})$ \\
\hline Graphite $^{\mathrm{a}}$ & \multirow{10}{*}{$1.46^{\mathrm{c}}$} & 0.3369 & 8.918 \\
\hline Graphite $(\varepsilon, \sigma)^{\mathrm{b}}$ & & 0.336 & 9.08 \\
\hline Virtual Graphite $(2 \varepsilon, \sigma)^{\mathrm{b}}$ & & 0.336 & 6.414 \\
\hline Virtual Graphite $(5 \varepsilon, \sigma)^{\mathrm{b}}$ & & 0.336 & 4.046 \\
\hline Virtual Graphite $(7 \varepsilon, \sigma)^{\mathrm{b}}$ & & 0.336 & 3.413 \\
\hline Virtual Graphite $(10 \varepsilon, \sigma)^{\mathrm{b}}$ & & 0.336 & 2.848 \\
\hline Virtual Graphite $(10 \varepsilon, 1.1 \sigma)^{\mathrm{b}}$ & & 0.3691 & 3.119 \\
\hline Virtual Graphite $(10 \varepsilon, 1.2 \sigma)^{\mathrm{b}}$ & & 0.4031 & 3.367 \\
\hline Virtual Graphite $(10 \varepsilon, 1.3 \sigma)^{\mathrm{b}}$ & & 0.4372 & 3.473 \\
\hline Virtual Graphite $(10 \varepsilon, 1.4 \sigma)^{\mathrm{b}}$ & & 0.4712 & 3.554 \\
\hline$h-\mathrm{BN}^{\mathrm{b}}$ & $1.29^{\mathrm{c}}$ & 0.3357 & 6.23 \\
\hline
\end{tabular}

\footnotetext{
${ }^{\mathrm{a}}$ modeled by AIREBO force field (Stuart et al., 2000)

${ }^{\mathrm{b}}$ modeled by Tersoff and LJ potentials (Kinacı et al., 2012; Tersoff, 1989)

${ }^{c}$ calculated using modified Tersoff potential for $h$-BN (Kinac1 et al., 2012; Singh et al., 2013)
}

\section{Discussions}

Before we draw conclusions, this section will provide some in-depth discussions and implications.

The mechanical buckling of anisotropic materials has been a topic of interests for a quite long time. The recent book from Bažant provides an excellent review on relevant topics (Bažant and Cedolin, 2010). Several different cases have been studied. The internal buckling happens for a continuum block/column under a uniaxial compression and being constrained by rigid and perfectly lubricated side edges (Biot, 1965). As the slenderness ratio approaching to zero, this case appears to be similar to our MD simulations. But it should be aware that previous models usually assumed the 
anisotropic materials to be incompressible. The incompressibility should inevitably bring the effects of the rigid side boundary to the buckling that happens in the internal part of the block. In order to directly compare with our theoretical model, we did some derivations of the Biot's original model (Biot, 1965). Indeed, the critical buckling strain $\varepsilon_{\mathrm{cr}}$ of such internal buckling, i.e., $4 C_{44} /\left(3 C_{11}-C_{13}\right)$ is quite different from our model $C_{44} / C_{11}$ that is derived from Eq. (12) in accordance to the fixed side boundary condition in MD. The second case studied previously is the buckling of a thick slab under a uniaxial compression with free side boundaries. But the materials were also assumed to be incompressible. As the slenderness ratio approaching to zero, Biot pointed out that it should coincide with the surface buckling of a semi-infinite half space, which clearly is different from our study. The third case is the buckling of a thick beam. As early as in 1889, Engesser proposed a model that took both the bending and shearing effect into account (Bažant and Cedolin, 2010). The critical buckling strain can be expressed as:

$$
{ }_{c r}=4^{2}\left[\left(\frac{L}{-}\right)^{2}+4^{2} \frac{Y}{G}\right]^{1},
$$

where $Y$ is Young's modulus of the column along longitudinal $x$ direction. Here $\eta$ is a factor related to the geometry of the cross-section (Timoshenko and Gere, 2012). It is usually taken 1.12 and 1.11 for rectangular and circular cross-sections, respectively. Taking the slenderness ratio $L / \rho$ approaching zero in Eq. (19) leads to a critical strain $\varepsilon_{\mathrm{cr}}=G / \eta Y$. It should be noted that the thick beam can deform in the transverse direction under the uniaxial compression, which is analogous to the freely relaxed side boundary condition we discussed in section 3 , i.e., $b_{0}=C_{13} / C_{33}$. The clear difference can be easily understood since a beam theory was used in Engesser's model that is not appropriate for a very thick three-dimension block. More importantly, it should be aware that previous homogeneous continuum mechanical models cannot explain the size effect, which is observed in our MD simulations and has been successfully explained using our model.

It is worth noting that our homogeneous continuum mechanical model Eq. (12) embodies neither an intrinsic length scale nor internal atomistic microstructures. The graphite column is simply described as a homogeneous continuum bulk. The IBS agrees very well with those determined by MD simulations (down to $20 \mathrm{~nm}$ ). This excellent agreement suggests that the critical buckling point of a thick graphite column ( zero slenderness ratio) above tens of nanometer scale is governed by its macroscopic elastic properties. This should be true for other hexagonal layered crystalline materials (Table IV). This conclusion is also consistent with previous studies of multi-walled carbon nanotubes 
(Liu et al., 2001; 2003), which modeled the multi-walled carbon nanotube (MWCNT) as a homogeneous continuum beam and successfully explained the rippling in MWCNT under bending in experiments (Poncharal et al., 1999). It should be noted that to model the post-buckling of the layered crystalline materials, such as the formation of kinking band, we may still require atomistic simulations (Li et al., 2007; Liu et al., 2011), thin shell/plate FEA models (Liu et al., 2005), or those atomistic-based FEA techniques (Arroyo and Belytschko, 2003).

From our homogeneous continuum mechanics model, we can conclude that the drastically low interlayer shear modulus $C_{44}$ in comparison with the in-plane modulus $C_{11}$ is the origin for the observed profound shear mode of instability and the anomalous elastic buckling of a column with an infinitesimal slenderness ratio. For a crystal material, the concept of elastic anisotropy degree $\delta(\boldsymbol{C})$ is often adopted to quantify the difference of elastic modulus along different crystalline directions (Nye, 1985; Wang and Zheng, 2007). The special crystal structure of a layered material implies a high $\delta(\boldsymbol{C})$. Indeed, among the top 20 hexagonal crystal materials with a high $\delta(\boldsymbol{C})$, most of them are layered crystalline materials (Wang and Zheng, 2007). It is natural to expect that a high elastic anisotropy degree $\delta(\boldsymbol{C})$ should lead to a small IBS $\varepsilon_{\mathrm{cr}}$. However, a comparison between the IBS $\varepsilon_{\mathrm{cr}}$ results and the anisotropy degree shows several exceptions. For instance, in Table IV, $\mathrm{MoS}_{2}$, Muscovite $\left[\mathrm{KAl}_{2} \mathrm{Si}_{3} \mathrm{O}_{10}(\mathrm{OH}, \mathrm{F})_{2}\right]$, or Rubidium nickel chloride $\left(\mathrm{RbNiCl}_{3}\right)$ has a similar IBS $\varepsilon_{\mathrm{cr}}$, i.e., 0.1583 , 0.1622 , and 0.1552 , but they show a significant difference in $\delta(\boldsymbol{C})$, i.e., 0.608, 0.5, and 0.408. Another example is that although Muscovite $\left[\mathrm{KAl}_{2} \mathrm{Si}_{3} \mathrm{O}_{10}(\mathrm{OH}, \mathrm{F})_{2}\right]$ has a larger $\delta(C)$ in comparison with Biotite $\left[\mathrm{K}(\mathrm{Mg}, \mathrm{Fe})_{3} \mathrm{AlSi}_{3} \mathrm{O}_{10}(\mathrm{OH}, \mathrm{F})_{2}\right]$, i.e., 0.608 vs. 0.557, its IBS $\varepsilon_{\mathrm{cr}}$ result is much higher as well, i.e., 0.1583 vs. 0.0643. Here we propose that the $\underset{\mathrm{cr}}{\mathrm{IBS}}[\mathrm{Eq}$. (12)] under the freely relaxed side boundary condition $\left(b_{0}=C_{13} / C_{33}\right)$ could serve as an alternative measure to characterize the degree of elastic anisotropy for hexagonal crystal materials. In the same spirit, Eq. (A13) and (A14) could be used to measure the degree of elastic anisotropy for orthorhombic crystalline materials. One clear advantage is that such a measure has a clearer physical meaning.

In light of the very weak interlayer physical interactions, it is intuitively reasonable to approximate the critical buckling stress/strain of a multi-layered stack by that of a mono-crystal-layer (Guo et al., 2011). Our study shows that such an approximation is problematic. For example, based on Euler model, for a graphene layer with length $L=20,30$, or $40 \mathrm{~nm}$, the $\varepsilon_{\mathrm{cr}}$ is $0.00358 \%, 0.00159 \%$, and $0.000896 \%$ given the thickness of graphene monolayer as $0.066 \mathrm{~nm}$ (Wang et al., 2005; 
Yakobson et al., 1996), But in our MD simulations for a multi-layer stack, they share a similar $\varepsilon_{\mathrm{cr}}=$ $0.0351 \%$. It clearly shows that despite its small magnitude, the interlayer modulus $C_{44}$ plays a decisive role in determining the mechanical buckling of a multi-layered stack of graphenes.

Employing elastic buckling to tune the physical properties of layered crystalline materials has several clear advantages. First, there are no chemical or physical damages to the crystal integrity. It could avoid some undesired side effects that often occur when tailoring the physical properties via methods such as cutting, chemical or physical adsorptions. Second, in principle, the elastic buckling is recoverable. That means utilizing buckling under a cyclic loading/unloading condition can repeatedly control the material morphologies and thus their properties. This is highly desirable in nanotechnology, which can enable many new applications, such as the mechanical sensor, and the responsive materials.

Making use of the shear mode of instability has several more advantages. First, a layered crystalline material can undergo a mechanical buckling with a very low slenderness ratio $(L / \rho \sim 0)$, suggesting that the buckling wavelength $L$ can be tuned to a very small value. Our MD simulations demonstrate the scale of $L$ down to $\sim 20 \mathrm{~nm}$. It can be used to generate periodic surface structures at a nanometer scale, which is a difficult task by employing the bending mode of instability. Second, a distinctive kinking morphology is the signature of the shear mode instability at post-buckling stage (Budiansky et al., 1998; Liu et al., 2010). In the kink, there is a sharp transition corner connecting two consecutive straight segments, which is potentially useful in some novel applications. For example, an electric current in graphene nano-bubbles can generate a giant pseudo-magnetic field (Levy et al., 2010). It was found that strength of the magnetic field depended on a change of curvature. The sharp corners in the kinks could be used to design nano-devices that can generate dynamically tunable giant pseudo-magnetic fields. Third, under the shear mode of instability, there are no strains in the basal planes of the layered crystalline materials (only shear deformation occurs among adjacent crystal layers). This could be another benefit, if the atomistic structure of crystal layers would like to be conserved.

\section{Summary}

In this paper, we study the elastic buckling of a column made of layered crystalline materials being subject to a uniaxial compressive load along the basal plane direction, using MD simulations 
and continuum mechanics modeling. In our MD simulations for graphite, $h$-BN, virtual graphite, and virtual $h$-BN, we found that the columns (at a zero slenderness ratio due to periodic boundary condition) had a constant critical buckling strain value given that the length $L$ is higher than certain values. Our continuum mechanics model reveals that such a converged critical bucking strain solely depends on the material's elastic constants (without structural dimensions), implying that it is an intrinsic material property. A new concept, intrinsic buckling strain (IBS), is thus defined. For a set of typical layered crystalline materials, the $\varepsilon_{\mathrm{cr}}^{\mathrm{IBS}}$ is much lower than elastic limit stain of the materials, indicating the mechanical buckling occurs prior to the failure of the materials. Our results also reveal that a high degree of elastic anisotropy is the origin for the anomalous mechanical buckling in the absence of structural slenderness.

In MD simulations, reducing the length $L$ leads to a gradual increase of $\varepsilon_{\mathrm{cr}}$, deviating from the constant $\varepsilon_{\mathrm{cr}}^{\mathrm{IBS}}$. We introduced a bending energy term, which describes the bending deformation of each individual atomic layer, in the continuum mechanics model. The good agreement between our continuum model [Eq. (18)] and the MD simulations indicates that the bending deformation of atomic layers is the origin for the observed size effect. A characteristic length parameter $l_{0}$ is defined, which is an intrinsic material parameter. When the column length $L$ is comparable or smaller than $l_{0}$, the size effect on $\varepsilon_{\mathrm{cr}}$ shows up.

Besides the layered crystalline materials, some biomaterials (e.g. nacre, shell, bone, etc.), fiber reinforce composites, or geological strata also have the similar microstructures and high elastic anisotropy. Our studies could provide some insights to understand their mechanical buckling behavior. In the end, some in-depth discussions and potential applications in nanotechnology are provided. This study could provide guidelines for engineering layered crystalline materials in various nano-materials and nano-devices via mechanical buckling.

\section{Appendix. Continuum mechanics model for intrinsic buckling strain of orthorhombic crystalline materials}

A continuum mechanics model is detailed here for a column made of orthorhombic crystalline materials [Fig. 1(b)]. The mechanical buckling could occur in either of the two lateral directions. Thus periodic boundary conditions are applied in both $y$ and $z$ directions. Similar to the previous derivations in section 3 , boundary conditions are 


$$
\begin{aligned}
& \left.u_{1}\right|_{x=L / 2}=12,\left.\quad 12\right|_{x=L / 2}=0,\left.\quad 13\right|_{x=L / 2}=0, \\
& \left.u_{1}\right|_{x=L / 2}=12,\left.\quad 12\right|_{x=L / 2}=0,\left.\quad 13\right|_{x=L / 2}=0, \\
& \left.u_{2}\right|_{x=L / 2}=\left.u_{2}\right|_{x=L / 2},\left.\quad u_{3}\right|_{x=L / 2}=\left.u_{3}\right|_{x=L / 2}
\end{aligned}
$$

where $\delta$ is the relative displacement of the both ends moving toward each other. The displacement fields can be expressed as

$$
u_{1}=x+{ }_{n=1}^{N} a_{n} \frac{L}{2 n} \sin \frac{2 n x}{L}, \quad u_{2}=k_{0} y+{ }_{n=1}^{N} k_{n} \frac{L}{2 n} \cos \frac{2 n x}{L}, \quad u_{3}=b_{0} z+{ }_{n=1}^{N} b_{n} \frac{L}{2 n} \cos \frac{2 n x}{L},
$$

where $\varepsilon=\delta / L$ represents the homogeneous compressive strain in $x$-direction prior to elastic buckling. Note that $u_{2}$ has a different expression compared to the transverse isotropic materials, e.g., hexagonal crystalline materials. The first terms of $u_{2}$ and $u_{3}$ are adopted to account for the different types of side boundary conditions in $z$ and $y$ directions, respectively. A fixed side boundary condition, as used in our MD simulations for graphite etc., means $k_{0}$ and $b_{0}$ equal to zero, whereas the freely relaxed side boundary condition requires $k_{0}=C_{12} / C_{22}$ and $b_{0}=C_{13} / C_{33}$ in which $C_{12}, C_{13}, C_{22}$ and $C_{33}$ are elastic constants. These displacement fields [Eq. (A2)] satisfy the boundary conditions [Eq. (A1)]. Parameters $a_{n}, k_{n}$ and $b_{n}$ are unknown coefficients. Only when the load is above the critical buckling value $\delta_{\text {cr }}$, the $a_{n}, k_{n}$ and $b_{n}$ will have nonzero solutions.

Deformation gradient $\boldsymbol{F}$ and the first Seth strain $\boldsymbol{E}$ can be derived based on the displacement fields as

$$
\begin{aligned}
& F_{11}=1+\frac{\partial u_{1}}{\partial x}, \quad F_{22}=1+\frac{\partial u_{2}}{\partial y}, \quad F_{33}=1+\frac{\partial u_{3}}{\partial z}, \\
& F_{12}=\frac{\partial u_{1}}{\partial y}, \quad F_{13}=\frac{\partial u_{1}}{\partial z}, \quad F_{23}=\frac{\partial u_{2}}{\partial z}, \\
& F_{21}=\frac{\partial u_{2}}{\partial x}, \quad F_{31}=\frac{\partial u_{3}}{\partial x}, \quad F_{32}=\frac{\partial u_{3}}{\partial y} \\
& E_{11}=\left(F_{11}^{2}+F_{21}^{2}+F_{31}^{2}-1\right) / 2, \quad E_{22}=\left(F_{12}^{2}+F_{22}^{2}+F_{32}^{2}-1\right) / 2, \quad E_{33}=\left(F_{13}^{2}+F_{23}^{2}+F_{33}^{2}-1\right) / 2 \\
& E_{12}=E_{21}=\left(F_{11} F_{12}+F_{21} F_{22}+F_{31} F_{32}\right) / 2, \quad E_{13}=E_{31}=\left(F_{11} F_{13}+F_{21} F_{23}+F_{31} F_{33}\right) / 2, \\
& E_{23}=E_{32}=\left(F_{12} F_{13}+F_{22} F_{23}+F_{32} F_{33}\right) / 2
\end{aligned}
$$

For an orthorhombic crystalline material, the constitutive law of linear elasticity can be expressed as 


$$
\left.\begin{array}{l}
{ }_{11} 2 \\
33 \\
23 \\
31 \\
12
\end{array}\right)=\left(\begin{array}{cccccc}
C_{11} & C_{12} & C_{13} & 0 & 0 & 0 \\
C_{12} & C_{22} & C_{23} & 0 & 0 & 0 \\
C_{13} & C_{23} & C_{33} & 0 & 0 & 0 \\
0 & 0 & 0 & C_{44} & 0 & 0 \\
0 & 0 & 0 & 0 & C_{55} & 0 \\
0 & 0 & 0 & 0 & 0 & C_{66}
\end{array}\right)\left(\begin{array}{c}
{ }_{11} \\
{ }_{22} \\
2_{23} \\
2_{31} \\
2_{12}
\end{array}\right),
$$

where $\sigma$ and $\tau$ represent the normal and shear stress components, $\varepsilon$ and $\gamma$ denote the normal and shear strain components, and $C_{i j}$ are the stiffness constants. Substituting Eq. (A4) into Eq. (A5) yields the second Piola-Kirchhoff stress $\boldsymbol{T}$ as

$$
\begin{aligned}
& T_{11}=C_{11} E_{11}+C_{12} E_{22}+C_{13} E_{33}, \quad T_{22}=C_{12} E_{11}+C_{22} E_{22}+C_{23} E_{33}, \\
& T_{33}=C_{13} E_{11}+C_{23} E_{22}+C_{33} E_{33}, \quad T_{23}=2 C_{44} E_{23}, \quad T_{13}=2 C_{55} E_{13}, \quad T_{12}=2 C_{66} E_{12} \text {. }
\end{aligned}
$$

Then the strain energy density $U$ is

$$
U=1 / 2\left(T_{11} E_{11}+T_{22} E_{22}+T_{33} E_{33}+2 T_{13} E_{13}+2 T_{23} E_{23}+2 T_{12} E_{12}\right) \text {. }
$$

Integrating the energy density $U$ in the column leads to the potential energy as

$$
W=\frac{1}{L} \int_{-L / 2}^{L / 2} U d x,
$$

in which unit lengths are taken along the $y$ and $z$ directions, owing to the periodic boundary conditions.

Following the principle of minimum total potential energy, partial derivatives of $W$ with respect to the undetermined coefficients $a_{n}, b_{n}$, and $k_{n}$ should be equal to zero. Thus,

$$
\begin{aligned}
& g_{1}=\partial \boldsymbol{W} / \partial b_{1}=b_{1}\left(C_{55}-\left(C_{11}-2 C_{55} b_{0}-C_{12} k_{0}-C_{13} b_{0}\right) \varepsilon\right) / 2+O\left(\varepsilon^{2}\right)=0 \\
& g_{2}=\partial \boldsymbol{W} / \partial b_{2}=b_{2}\left(C_{55}-\left(C_{11}-2 C_{55} b_{0}-C_{12} k_{0}-C_{13} b_{0}\right) \varepsilon\right) / 2+O\left(\varepsilon^{2}\right)=0 \\
& \ldots \ldots \\
& m_{1}=\partial \boldsymbol{W} / \partial k_{1}=k_{1}\left(C_{66}-\left(C_{11}-2 C_{66} k_{0}-C_{12} k_{0}-C_{13} b_{0}\right) \varepsilon\right) / 2+O\left(\varepsilon^{2}\right)=0 \\
& m_{2}=\partial \boldsymbol{W} / \partial k_{2}=k_{2}\left(C_{66}-\left(C_{11}-2 C_{66} k_{0}-C_{12} k_{0}-C_{13} b_{0}\right) \varepsilon\right) / 2+O\left(\varepsilon^{2}\right)=0, \\
& \ldots \ldots \\
& f_{1}=\partial \boldsymbol{W} / \partial a_{1}=a_{1}\left(C_{11}-\left(3 C_{11}-C_{12} k_{0}-C_{13} b_{0}\right) \varepsilon\right) / 2+O\left(\varepsilon^{2}\right)=0 \\
& f_{2}=\partial \boldsymbol{W} / \partial a_{2}=a_{2}\left(C_{11}-\left(3 C_{11}-C_{12} k_{0}-C_{13} b_{0}\right) \varepsilon\right) / 2+O\left(\varepsilon^{2}\right)=0
\end{aligned}
$$

where the quadratic and higher order terms of strain $\varepsilon$ are omitted. Note that derivatives with respect to $b_{n}(n=1,2,3,4 \ldots)$ always yield the same equation as 


$$
C_{55}-\left(C_{11}-2 C_{55} b_{0}-C_{12} k_{0}-C_{13} b_{0}\right) \varepsilon=0 \text {. }
$$

The derivatives with respect to $k_{n}(n=1,2,3,4 \ldots)$ lead to an equation as

$$
C_{66}-\left(C_{11}-2 C_{66} k_{0}-C_{12} k_{0}-C_{13} b_{0}\right) \varepsilon=0 .
$$

The derivatives with respect to $a_{n}(n=1,2,3,4 \ldots)$ lead to an equation as

$$
C_{11}-\left(3 C_{11}-C_{12} k_{0}-C_{13} b_{0}\right) \varepsilon=0 .
$$

From Eq. (A10), (A11) or (A12), we obtained the IBS $\varepsilon_{\mathrm{cr}}$ as

$$
\varepsilon_{c r}=\frac{C_{55}}{C_{11}-C_{12} k_{0}-\left(2 C_{55}+C_{13}\right) b_{0}},
$$

or

$$
\varepsilon_{c r}=\frac{C_{66}}{C_{11}-C_{13} b_{0}-\left(2 C_{66}+C_{12}\right) k_{0}},
$$

or

$$
\varepsilon_{c r}=\frac{C_{11}}{3 C_{11}-C_{12} k_{0}-C_{13} b_{0}} .
$$

Knowledge of the perovskite layered crystalline materials is quite limited (Geim and Grigorieva, 2013). A complete set of elastic constants for $\mathrm{LaNb}_{2} \mathrm{O}_{7},(\mathrm{Ca}, \mathrm{Sr})_{2} \mathrm{Nb}_{3} \mathrm{O}_{10}, \mathrm{Bi}_{4} \mathrm{Ti}_{3} \mathrm{O}_{12}, \mathrm{Ca}_{2} \mathrm{Ta}_{2} \mathrm{TiO}_{10}$ are not available. Therefore, a quantitative comparison between the theoretical results Eq. (A13) and (A14) and numerical simulations are not feasible at present.

Note that Eq. (A13) and Eq. (A15) can be reduced into Eq. (12) and (13) for the hexagonal crystalline materials, through letting $C_{22}=C_{11}, C_{23}=C_{13}$, and $C_{55}=C_{44}$ because of the hexagonal crystal symmetry and $k_{0}=0$ due to the plane-strain conditions adopted in Eq. (12) and (13).

\section{Acknowledgement}

J. Z. Liu acknowledges the support of a seed grant from the engineering faculty at Monash University and acknowledges National Computational Infrastructure at Australian National University and Monash Sun Grid high-performance computing facility at Monash University for providing the computational resource. Y. L. acknowledges the financial support from NSFC through Grant No. 11302163 and 11321062, and Q. S. Z. acknowledges the financial support from NSFC through Grant No. 10832005, the National Basic Research Program of China (Grant No. 2007CB936803), and the 
National 863 Project (Grant No. 2008AA03Z302).

\section{Reference}

Arroyo, M., Belytschko, T., 2003. Nonlinear Mechanical Response and Rippling of Thick Multiwalled Carbon Nanotubes. Phys. Rev. Lett. 91, 215505. doi:10.1103/PhysRevLett.91.215505

Bažant, Z.P., Cedolin, L., 2010. Stability of Structures. World Scientific.

Biot, M.A., 1965. Mechanics of Incremental Deformations, 1965. John Wiley \& Sons Ltd, New York.

Biot, M.A., 1957. Folding Instability of a Layered Viscoelastic Medium under Compression. Proceedings of the Royal Society A: Mathematical, Physical and Engineering Sciences 242, 444-454. doi:10.1098/rspa.1957.0187

Bowden, N., Brittain, S., Evans, A.G., Hutchinson, J.W., Whitesides, G.M., 1998. Spontaneous formation of ordered structures in thin films of metals supported on an elastomeric polymer. Nature 393, 146-149.

Brau, F., Vandeparre, H., Sabbah, A., Poulard, C., Boudaoud, A., Damman, P., 2010. Multiple-length-scale elastic instability mimicsparametric resonance of nonlinear oscillators. Nat. Photo. 7, 56-60. doi:10.1038/nphys1806

Budd, C.J., Edmunds, R., Hunt, G.W., 2003. A nonlinear model for parallel folding with friction. Proceedings of the Royal Society A: Mathematical, Physical and Engineering Sciences 459, 2097-2119. doi:10.1098/rspa.2003.1139

Budiansky, B., Fleck, N.A., Amazigo, J.C., 1998. On kink-band propagation in fiber composites. J. Mech. Phys. Solids 46, 1637-1653.

Byskov, E., Christoffersen, J., Christensen, C.D., Poulsen, J.S., 2002. Kinkband formation in wood and fiber composites - morphology and analysis. Int. J. Solids Structures 39, 3649-3673.

Ci, L., Song, L., Jin, C., Jariwala, D., Wu, D., Li, Y., Srivastava, A., Wang, Z.F., Storr, K., Balicas, L., Liu, F., Ajayan, P.M., 2010. Atomic layers of hybridized boron nitride and graphene domains. Nat. Mat. 9, 430-435. doi:10.1038/nmat2711

Ci, L., Xu, Z., Wang, L., Gao, W., Ding, F., Kelly, K., Yakobson, B., Ajayan, P., 2008. Controlled nanocutting of graphene. Nano Res. 1, 116-122.

Cranford, S.W., 2013. Buckling induced delamination of graphene composites through hybrid molecular modeling. Appl. Phys. Lett. 102, 031902-6. doi:10.1063/1.4788734

Duclaux, L., Nysten, B., Issi, J.P., Moore, A.W., 1992. Structure and low-temperature thermal conductivity of pyrolytic boron nitride. Phys. Rev. B 46, 3362-3367. doi:10.1103/PhysRevB.46.3362

Efimenko, K., Rackaitis, M., Manias, E., Vaziri, A., Mahadevan, L., Genzer, J., 2005. Nested self-similar wrinkling patterns in skins. Nat. Mat. 4, 293-297. doi:10.1038/nmat1342

Elias, D.C., Nair, R.R., Mohiuddin, T., Morozov, S.V., 2009. Control of graphene's properties by reversible hydrogenation: evidence for graphane. Science 323, 610-613. doi:10.1126/science.1092905)

Geim, A., Novoselov, K., 2007. The rise of graphene. Nat. Mat. 6, 183-191.

Geim, A.K., Grigorieva, I.V., 2013. Van der Waals heterostructures. Nature 499, 419-425. doi:10.1038/nature12385

Gere, J.M., Timoshenko, S.P., 1998. Mechanics of Materials; 4th ed. Chapman and Hall, London.

Ghosh, S., Arroyo, M., 2013. An atomistic-based foliation model for multilayer graphene materials and nanotubes. Journal of the Mechanics and Physics of Solids. doi:10.1016/j.jmps.2012.07.002

Golberg, D., Bando, Y., Huang, Y., Terao, T., Mitome, M., 2010. Boron nitride nanotubes and nanosheets. ACS Nano 4, 2979-2993. doi:10.1038/nmat3542

Gould, T., LIU, Z., Liu, J.Z., Dobson, J.F., Zheng, Q.-S., 2013. Binding and interlayer force in the near-contact region of two graphite slabs: Experiment and theory. J. Chem. Phys. doi:10.1063/1.4839615

Guo, F., Kim, F., Han, T.H., Shenoy, V.B., Huang, J., Hurt, R.H., 2011. Hydration-Responsive Folding and 
Unfolding in Graphene Oxide Liquid Crystal Phases. ACS Nano 5, 8019-8025. doi:10.1021/nn2025644

Haigh, S.J., Gholinia, A., Jalil, R., Romani, S., Britnell, L., Elias, D.C., Novoselov, K.S., Ponomarenko, L.A., Geim,

A.K., Gorbachev, R., 2012. Cross-sectional imaging of individual layers and buried interfaces of graphene-based heterostructures and superlattices. Nat. Mat. 11, 1-4. doi:10.1038/nmat3386

Hohlfeld, E., Mahadevan, L., 2011. Unfolding the Sulcus. Phys. Rev. Lett. 106, 105702. doi:10.1103/PhysRevLett.106.105702

Huang, Z.Y., Hong, W., Suo, Z., 2005. Nonlinear analyses of wrinkles in a film bonded to a compliant substrate. Journal of the Mechanics and Physics of Solids 53, 2101-2118. doi:10.1016/j.jmps.2005.03.007

Hunt, G.W., Peletier, M.A., Wadee, M.A., 2000. The Maxwell stability criterion in pseudo-energy models of kink banding. Journal of Structural Geology 22, 669-681.

Kelly, B.T., 1981. Physics of Graphite, 1st ed. Applied Science Publishers, London.

Kim, D.H., Ahn, J.H., Choi, W.M., Kim, H.S., Kim, T.H., Song, J., 2008. Stretchable and foldable silicon integrated circuits. Science 320, 507-511. doi:10.1126/science.1078727

Kim, J., Yoon, J., Hayward, R.C., 2009. Dynamic display of biomolecular patterns through an elastic creasing instability of stimuli-responsive hydrogels. Nat. Mat. 9, 159-164. doi:10.1038/nmat2606

Kim, P., Abkarian, M., Stone, H.A., 2011. Hierarchical folding of elastic membranes under biaxial compressive stress. Nat. Mat. 10, 952-957. doi:10.1038/nmat3144

Kim, R.H., Kim, D.H., Xiao, J., Kim, B.H., Park, S.I., Panilaitis, B., Ghaffari, R., Yao, J., Li, M., Liu, Z., Malyarchuk, V., Kim, D.G., Le, A.-P., Nuzzo, R.G., Kaplan, D.L., Omenetto, F.G., Huang, Y., Kang, Z., Rogers, J.A., 2010. Waterproof AlInGaP optoelectronics on stretchable substrates with applications in biomedicine and robotics. Nat. Mat. 9, 929-937. doi:10.1038/nmat2879

Koo, W.H., Jeong, S.M., Araoka, F., Ishikawa, K., Nishimura, S., Toyooka, T., Takezoe, H., 2010. Light extraction from organic light-emitting diodesenhanced by spontaneously formed buckles. Nat. Photo. 4, 222-226. doi:10.1038/nphoton.2010.7

Kyriakides, S., Arseculeratne, R., Perry, E.J., Liechti, K.M., 1995. On the compressive failure of fiber reinforced composites. Int. J. Solids Structures 32, 689-738.

Kınacı, A., Haskins, J.B., Sevik, C., Çağın, T., 2012. Thermal conductivity of BN-C nanostructures. Phys. Rev. B 86, 115410-8. doi:10.1103/PhysRevB.86.115410

Leahy, B.D., Pocivavsek, L., Meron, M., Lam, K.L., Salas, D., Viccaro, P.J., Lee, K.Y.C., Lin, B., 2010. Geometric Stability and Elastic Response of a Supported Nanoparticle Film. Phys. Rev. Lett. 105, 058301. doi:10.1103/PhysRevLett.105.058301

Lebègue, S., Harl, J., Gould, T., Ángyán, J., Kresse, G., Dobson, J., 2010. Cohesive Properties and Asymptotics of the Dispersion Interaction in Graphite by the Random Phase Approximation. Phys. Rev. Lett. 105, 196401. doi:10.1103/PhysRevLett.105.196401

Lee, C., Wei, X., Kysar, J.W., Hone, J., 2008. Measurement of the Elastic Properties and Intrinsic Strength of Monolayer Graphene. Science 321, 385-388. doi:10.1126/science.1157996

Levy, N., Burke, S.A., Meaker, K.L., Panlasigui, M., Zettl, A., Guinea, F., Neto, A.H.C., Crommie, M.F., 2010.

Strain-Induced Pseudo-Magnetic Fields Greater Than 300 Tesla in Graphene Nanobubbles. Science 329, 544-547. doi:10.1126/science.1191700

Li, X., Yang, W., Liu, B., 2007. Bending Induced Rippling and Twisting of Multiwalled Carbon Nanotubes. Phys. Rev. Lett. 98, 205502. doi:10.1103/PhysRevLett.98.205502

Lindberg, H.E., 2003. Little Book of Dynamic Buckling. LCE Science/Software.

Liu, J.Z., 2002. Studies on Several Mechanical Problems of Carbon Nanotubes. Tsinghua University, Beijing.

Liu, J.Z., Zheng, Q., Jiang, Q., 2003. Effect of bending instabilities on the measurements of mechanical properties of multiwalled carbon nanotubes. Phys. Rev. B 67, 075414. doi:10.1103/PhysRevB.67.075414

Liu, J.Z., Zheng, Q., Jiang, Q., 2001. Effect of a Rippling Mode on Resonances of Carbon Nanotubes. Phys. Rev. 
Lett. 86, 4843-4846. doi:10.1103/PhysRevLett.86.4843

Liu, J.Z., Zheng, Q.-S., Wang, L.-F., Jiang, Q., 2005. Mechanical properties of single-walled carbon nanotube bundles as bulk materials. Journal of the Mechanics and Physics of Solids 53, 123-142. doi:10.1016/j.jmps.2004.06.008

Liu, Y., Xu, Z., Zheng, Q., 2011. Journal of the Mechanics and Physics of Solids. Journal of the Mechanics and Physics of Solids 59, 1613-1622. doi:10.1016/j.jmps.2011.04.014

Liu, Z., Liu, J.Z., Cheng, Y., Li, Z., Li, Z., Wang, L., Zheng, Q., 2012. Interlayer binding energy of graphite: A mesoscopic determination from deformation. Phys. Rev. B 85, 205418. doi:10.1103/PhysRevB.85.205418

Liu, Z., Zheng, Q.-S., Liu, J.Z., 2010. Stripe/kink microstructures formed in mechanical peeling of highly orientated pyrolytic graphite. Appl. Phys. Lett. 96, 201909. doi:10.1063/1.3422484

Malko, D., Neiss, C., Viñes, F., Görling, A., 2012. Competition for Graphene: Graphynes with Direction-Dependent Dirac Cones. Phys. Rev. Lett. 108, 086804. doi:10.1103/PhysRevLett.108.086804

Mounet, N., Marzari, N., 2005. First-principles determination of the structural, vibrational and thermodynamic properties of diamond, graphite, and derivatives. Phys. Rev. B 71, 205214-14. doi:10.1103/PhysRevB.71.205214

Nair, R.R., Ren, W., Jalil, R., Riaz, I., Kravets, V.G., Britnell, L., Blake, P., Schedin, F., Mayorov, A.S., Yuan, S., Katsnelson, M.I., Cheng, H.M., Strupinski, W., Bulusheva, L.G., Okotrub, A.V., Grigorieva, I.V., Grigorenko, A.N., Novoselov, K.S., Geim, A.K., 2010. Fluorographene: A Two-Dimensional Counterpart of Teflon. Small 6, 2877-2884. doi:10.1002/smll.201001555

Nicolosi, V., Chhowalla, M., Kanatzidis, M.G., Strano, M.S., Coleman, J.N., 2013. Liquid Exfoliation of Layered Materials. Science 340, 1226419. doi:10.1126/science.1226419

Novoselov, K.S., Geim, A.K., Morozov, S.V., Jiang, D., Zhang, Y., Dubonos, S.V., Grigorieva, I.V., Firsov, A.A., 2004. Electric Field Effect in Atomically Thin Carbon Films. Science 306, 666-669.

doi:10.1126/science. 1102896

Nye, J.F., 1985. Physical properties of crystals. Oxford University Press, New York.

Plimpton, S., 1995. Fast parallel algorithms for short-range molecular dynamics. Journal of Computational Physics 117, 1-19. doi:10.1006/jcph.1995.1039

Pocivavsek, L., Dellsy, R., Kern, A., Johnson, S., Lin, B., Lee, K.Y.C., Cerda, E., 2008. Stress and Fold Localization in Thin Elastic Membranes. Science 320, 912-916. doi:10.1126/science.1154069

Poncharal, P., Wang, Z.L., Ugarte, D., de Heer, W.A., 1999. Electrostatic Deflections and Electromechanical Resonances of Carbon Nanotubes. Science 283, 1513-1516. doi:10.1126/science.283.5407.1513

Price, N.J., Cosgrove, J.W., 1990. Analysis of Geological Structures. Cambridge University Press.

Qiu, L., Liu, J.Z., Chang, S.L.Y., Wu, Y., Li, D., 2012. Biomimetic superelastic graphene-based cellular monoliths. Nat. Comms. 3, 1241. doi:10.1038/ncomms 2251

Ren, M., Liu, Z., Zheng, Q.-S., Liu, J.Z., 2015. Mechanical buckling induced periodic kinking/stripe microstructures in mechanically peeled graphite flakes from HOPG. Acta Mech Sin 1-6. doi:10.1007/s10409-015-0417-6

Rogers, J.A., Someya, T., Huang, Y., 2010. Materials and Mechanics for Stretchable Electronics. Science 327, 1603-1607. doi:10.1126/science. 1182383

Schedin, F., Geim, A.K., Morozov, S.V., Hill, E.W., Blake, P., Katsnelson, M.I., Novoselov, K.S., 2007. Detection of individual gas molecules adsorbed on graphene. Nat. Mat. 6, 652-655. doi:10.1038/nmat1967

Singh, S.K., Neek-Amal, M., Costamagna, S., Peeters, F.M., 2013. Thermomechanical properties of a single hexagonal boron nitride sheet. Phys. Rev. B 87, 184106-7. doi:10.1103/PhysRevB.87.184106

Song, L., Ci, L., Lu, H., Sorokin, P.B., Jin, C., Ni, J., Kvashnin, A.G., Kvashnin, D.G., Lou, J., Yakobson, B.I., Ajayan, P.M., 2010. Large Scale Growth and Characterization of Atomic Hexagonal Boron Nitride Layers. Nano Lett. 10, 3209-3215. doi:10.1021/nl1022139 
Sorella, S., Spanu, L., Galli, G., 2009. Nature and Strength of Interlayer Binding in Graphite. Phys. Rev. Lett. 103, 196401. doi:10.1103/PhysRevLett.103.196401

Stafford, C.M., Harrison, C., Beers, K.L., Karim, A., Amis, E.J., VanLandingham, M.R., Kim, H.-C., Volksen, W., Miller, R.D., Simonyi, E.E., 2004. A buckling-based metrology for measuring the elastic moduli of polymeric thin films. Nat. Mat. 3, 545-550. doi:10.1038/nmat1175

Stuart, S.J., Tutein, A.B., Harrison, J.A., 2000. A reactive potential for hydrocarbons with intermolecular interactions. J. Chem. Phys. 112, 6472.

Tan, P.H., 2012a. The shear mode of multilayer graphene. Nat. Mat. 11, 294-300. doi:10.1038/nmat3245

Tan, P.H., 2012b. The shear mode of multilayer graphene. Nat. Mat. 11, 294-300. doi:10.1038/nmat3245

Tersoff, J., 1989. Modeling solid-state chemistry: Interatomic potentials for multicomponent systems. Phys. Rev. B 39, 5566-5568.

Timoshenko, S.P., Gere, J.M., 2012. Theory of Elastic Stability. Courier Dover Publications.

Vogt, P., De Padova, P., Quaresima, C., Avila, J., Frantzeskakis, E., Asensio, M.C., Resta, A., Ealet, B., Le Lay, G., 2012. Silicene: Compelling Experimental Evidence for Graphenelike Two-Dimensional Silicon. Phys. Rev.

Lett. 108, 155501. doi:10.1103/PhysRevLett.108.155501

Volkova, E.I., Jones, I.A., Brooks, R., Zhu, Y., Bichoutskaia, E., 2012. Sequential multiscale modelling of SiC/Al nanocomposites reinforced with WS_\{2\} nanoparticles under static loading. Phys. Rev. B 86, 104111. doi:10.1103/PhysRevB.86.104111

Wadee, M.A., Hunt, G.W., Peletier, M.A., 2004. Kink band instability in layered structures. Journal of the Mechanics and Physics of Solids 52, 1071-1091. doi:10.1016/j.jmps.2003.09.026

Wang, L., Zheng, Q., Liu, J.Z., Jiang, Q., 2005. Size Dependence of the Thin-Shell Model for Carbon Nanotubes. Phys. Rev. Lett. 95, 105501. doi:10.1103/PhysRevLett.95.105501

Wang, L.-F., Zheng, Q.-S., 2007. Extreme anisotropy of graphite and single-walled carbon nanotube bundles. Appl. Phys. Lett. 90, 153113. doi:10.1063/1.2722057

Wang, Q.H., Kalantar-Zadeh, K., Kis, A., Coleman, J.N., 2012. Electronics and optoelectronics of two-dimensional transition metal dichalcogenides. Nature 7, 699-712. doi:10.1038/nnano.2012.193

Wang, Y., Yang, R., Shi, Z., Zhang, L., Shi, D., Wang, E., Zhang, G., 2011. Super-Elastic Graphene Ripples for Flexible Strain Sensors. ACS Nano 5, 3645-3650. doi:10.1021/nn103523t

Wilson, J.A., Yoffe, A.D., 1969. The transition metal dichalcogenides discussion and interpretation of the observed optical, electrical and structural properties. Advances in Physics 18, 193-335. doi:10.1080/00018736900101307

Xu, Z., Xue, K., Xue, K., 2009. Engineering graphene by oxidation: a first-principles study. Nanotechnology 21, 045704. doi:10.1088/0957-4484/21/4/045704

Yakobson, B.I., Brabec, C.J., Bernholc, J., 1996. Nanomechanics of carbon tubes: instabilities beyond linear response. Phys. Rev. Lett.

Yu, M.F., 2004. Fundamental mechanical properties of carbon nanotubes: current understanding and the related experimental studies. Journal of engineering materials and technology 126, 271. doi:10.1115/1.1755245

Zang, J., Ryu, S., Pugno, N., Wang, Q., Tu, Q., Buehler, M.J., Zhao, X., 2013. Multifunctionality and control of the crumpling and unfolding of large-area graphene. Nat. Mat. 12, 1-5. doi:10.1038/nmat3542

Zartman, J.J., Shvartsman, S.Y., 2010. Unit Operations of Tissue Development: Epithelial Folding. Annu. Rev. Chem. Biomol. Eng. 1, 231-246. doi:10.1146/annurev-chembioeng-073009-100919 